NBSIR 82-2519

\title{
Rural and Non-Rural Civilian Residential Fire Fatalities in Twelve States
}

U.S. DEPARTMENT OF COMMERCE

National Bureau of Standards

National Engineering Laboratory

Center for Fire Research

Washington, DC 20234

June 1982

Sponsored in part by:

Federal Emergency Management Agency

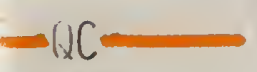

National Fire Data Center

1uv U.S. Fire Administration

.456

Washington, DC 20472 


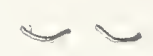




\section{RURAL AND NON-RURAL CIVILIAN RESIDENTIAL FIRE FATALITIES IN TWELVE STATES}

A. Gomberg and L. P. Clark

U.S. DEPARTMENT OF COMMERCE

National Bureau of Standards

National Engineering Laboratory

Center for Fire Research

Washington, DC 20234

June 1982

Sponsored in part by:

Federal Emergency Management Agency

National Fire Data Center

U.S. Fire Administration

Washington, DC 20472

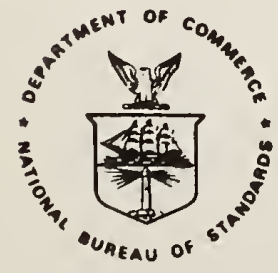

U.S. DEPARTMENT OF COMMERCE, Malcolm Baldrige, Secretary NATIONAL BUREAU OF STANDARDS, Ernest Ambler, Director 

IIST OF FIGURES . . . . . . . . . . . . . . . . . . . . . . iv

LIST OF TABLES . . . . . . . . . . . . . . . . . . . . . . . . v

Abstract . . . . . . . . . . . . . . . . . . . . . . I

1. INTRODUCTION . . . . . . . . . . . . . . . . . . . . . 1

2. POPULATION ESTIMATES . . . . . . . . . . . . . . . . . . . 4

2.1 Population of Area where Fatality Occurred . . . . . . . . . . . . 4 4

2.2 Estimated Populations ................... 5

3. OVERALl FAtALITY RATES . . . . . . . . . . . . . . . . . . . 6

4. General CAUSAL PATterns . . . . . . . . . . . . . . . . . . . . . . . 8

4.1 Fatality Rates by Cause . . . . . . . . . . . . . . . . 8

4.2 Type of fuel in Heating fires . . . . . . . . . . . . . . . . . 11

5. FIRE FATALITY SCENARIOS . . . . . . . . . . . . . . . . . . . 12

5.1 Heating Fire Scenarios . . . . . . . . . . . . . . . 12

5.2 Cooking Fire Scenarios . . . . . . . . . . . . . . . . . . . . 19

5.3 Smoking Fire Scenarios . . . . . . . . . . . . . . . . . . . . 21

5.4 Electrical Distribution Fire Scenarios . . . . . . . . . . . . . 22

5.5 Open Flame Fire Scenarios . . . . . . . . . . . . . . 26

6. SELECTED GENERAL OBSERVATIONS . . . . . . . . . . . . . . . . . . . 27

6.1 RURAL and NON-RURAL Race Comparisons . . . . . . . . . . . . . . . . . 27

6.2 Condition before Injury .. . . . . . . . . . . . . . . . . . 29

6.3 Property Type ........................... . 31

6.4 Smoke Detector Presence .. . . . . . . . . . . . . . . . . . 32

6.5 Fire Department Response .................... 33

7. CONCLUSions . . . . . . . . . . . . . . . . . . . . . 36

8. ACKNOWLEDGEMENT . . . . . . . . . . . . . . . . . . . . . 37

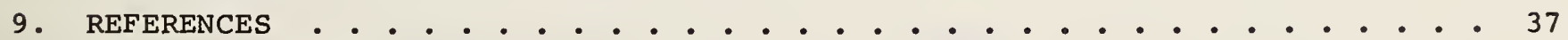

APPENDIX A. CODING STRUCTURES . . . . . . . . . . . . . . . . . . 38

APPENDIX B. CALCULATION OF POPULATIONS BY POPULATION INTERVAL • • • • • • • 45 


\section{LIST OF FIGURES}

Page

Figure 1. Residential fatality rates - ALL states . . . . . . . . . . 7

Figure 2. Fatality rates by cause for population intervals . . . . . . . . 9

Figure 3. Fatality rates by population interval for each cause . . . . . . 10

Figure 4. Fatality rates by cause for HIGH and LOW states. . . . . . . . 10

Figure 5. Fatalities by heating equipment and fuel . . . . . . . . . . . . 11

Figure 6. Fatality rates by race . . . . . . . . . . . . . . . . 28

Figure 7. Fatality rates by race/population interval . . . . . . . . . 28 
Table 1. Civilian, residential fire death totals and fire death rates for HIGH and LOW states

Table 2. Population estimates HIGH states . . . . . . . . . . . . . . . . . 5

Table 3. Population estimates Low rate states . . . . . . . . . . . . . . 5

Table 4. Fatality rates by population interval - HIGH rate states . . . . . 6

Table 5. Fatality rates by population interval - Low rate states . . . . ... 6

Table 6. Fatality rates by population interval - ALI states . . . . . . . 7

Table 7. RURAL and NON-RURAL fatality rates . . . . . . . . . . . . . . 8

Table 8. Fatality rates by cause . . . . . . . . . . . . . . . . . . . 9

Table 9. Heating fire fatality rates - ALL states (Form of material ignited; walls, ceiling, framing, insulation, flooring . . . . . . 13

Table 10. Heating fire fatality rates - ALL states (Form of material ignited: furniture, bedding, soft goods, trash) . . . . . . . . . . 13

Table 11. Heating fire fatality rates - ALL states (Form of material ignited: garment on person) . . . . . . . . . . . . . . . . . 13

Table 12. Heating fire fatality rates - ALL states (Form of material ignited: flammable liquid, fuel) : . . . . . . . . . . . . . . .

Table 13. Heating fire fatality rates - HIGH/LOW states (Form of material ignited: walls, ceiling, framing, insulation, flooring) . . . . . .

Table 14. Heating fire fatality rates - HIGH/LOW states (Form of material ignited: furniture, bedding, soft goods, trash) . . . . . . . . . .

Table 15. Heating fire fatality rates - HIGH/LOW states (Form of material ignited: garment on person) . . . . . . . . . . . . . . . . . .

Table 16. Heating fire fatality rates - HIGH/LOW states (Form of material ignited: flammable liquid, fuel

Table 17. Heating fire fatality rates ranked by equipment and area category .

Table 18. Cooking fire fatality rates - ALI states (Form of heat: all

(75\% gas fueled equipment)) . . . . . . . . . . . . . . . .

Table 19. Cooking fire fatality rates - HIGH/LOW states (Form of heat: all (75\% gas fueled equipment)) . . . . . . . . . . . . . . . . 19

Table 20. Smoking fire fatality rates - ALL states (Cause: smoking) . . . . . 21

Table 21. Smoking fire fatality rates - HIGH/LOW states (Cause: smoking) • . . 22

Table 22. Electrical distribution fire fatality rates - ALL states (Fixed property use: one and two family)

Table 23. Electrical distribution fire fatality rates - ALI states (Fixed property use: mobile homes)

Table 24. Electrical distribution fire fatality rates - ALL states (Fixed property use: other residential) 
Table 25. Electrical distribution fire fatality rates - HIGH/LOW states

(Fixed property use: one and two family) . . . . . . . . . . 24

Table 26. Electrical distribution fire fatality rates - HIGH/LOW states

(Fixed property use: mobile home) . . . . . . . . . . . . 24

Table 27. Electrical distribution fire fatality rates - HIGH/LOW states

(Fixed property use: other residential) . . . . . . . . . 25

Table 28. Open flame fire fatality rates - ALL states (Cause: open flame) . . 26

Table 29. Open flame fire fatality rates - HIGH/LOW states

(Cause: open flame)............................ 26

Table 30. Condition before injury - ALl states . . . . . . . . . . . . . 29

Table 31. Condition before injury - HIGH/LOW states . . . . . . . . . . . 30

Table 32. Property type - ALl states . . . . . . . . . . . . . . . 31

Table 33. Property type - HIGH/LOW states . . . . . . . . . . . . . 32

Table 34. Smoke detector presence - All states . . . . . . . . . . . . . 32

Table 35. Smoke detector presence - HIGH/LOW states . . . . . . . . . . . . 33

Table 36. Reported and unreported fatalities - HIGH/LOW states . . . . . . 34

Table 37. Unreported fatalities - HIGH states . . . . . . . . . . . . . 34

Table 38. Unreported fatalities by cause . . . . . . . . . . . . . . 35

Table 39. Unreported fatalities by form of heat of ignition . . . . . . . . 36 


\title{
RURAL AND NON-RURAL CIVILIAN RESIDENTIAL FIRE FATALITIES IN TWELVE STATES
}

\author{
A. Gomberg and Louis P. Clark
}

\section{Abstract}

The results of an analysis of fire causal factors in over 1600 fire fatalities are presented. The primary emphasis is on the identification of fire causes leading to demonstrated high fatality rates in rural areas. It was found that the most significant rural fire fatality cause was heating equipment, with improper installation and misuse of solid fueled heating equipment predominating. Other fire causes making significant contributions to high rural fatality rates were alsc investigated and documented. Additional data are currently being collected to enable further evaluation of rural fire problems.

Key Words: Residential fires; fire fatalities; fire cause; fire data; fire statistics; heating equipment; rural fires.

\section{INTRODUCTION}

In December 1978 the U.S. Fire Administration (USFA) published "Fire in the United States" [1] which reported a wide variaticn among the fire-death rates in the fifty states. The states with the highest reported death rates were Alaska, Maine, and a group of southern states: Oklahoma, Arkansas, Louisiana, Tennessee, Nississippi, Alabama, Georgia, and North and South Carolina. In "Highlights of Fire in the United States", Second Edition [2], the USFA again found higher than average fire-death rates in the scuthern and southeastern states and Alaska and the District of Columbia. These reports also indicate that the fire death problem is especially serious in rural communities. The National Bureau of standards (NBS), Center for Fire Research (CFR) designed a project for the USFA in which information on fire fatalities was collected and analyzed in order to provide insight into two questions raised by the previous studies:

1. Why is the fire-death rate in the southern and southeastern United States higher than in the rest of the United States?

2. What are the differences in fire death characteristics and causes between rural and non-rural populations?

This report is concerned with the second question, and addresses the differences between rural and non-rural fatality characteristics for civilian (non-fire service) residential fatalities.

In order to gain insight into these questions, data on 1,797 fire fatalities from twelve states were collected, including 1,606 civilian, residential fatalities. Based on data from earlier years six of these states were grouped as high death rate states: Mississippi, Alabama, Arkansas, Tennessee, Georgia, and Oklahoma; six of these states were grouped as low death rate states: Connecticut, Utah, Wisconsin, California, Florida, and Delaware. Each state contains both rural and non-rural populations. The data collected were used for the RURAL/NON-RURAL analyses which are the subject of this report. Differences between RURAI and NON-RURAL fatality rates across all 12 states as a group are presented, and where appropriate, further categorization of these rates by high and low death rate states is discussed.

\footnotetext{
Numbers in brackets indicate the literature references at the end of this paper.
} 
In each of the participating states the data were collected through the state fire marshal's office or a comparable office responsible for data collection. In each of the twelve states, a full one year collection period was agreed upon ${ }^{2}$. Then a report was obtained for every fatality which could be identified as being the result of a fire which occurred in that state over the one year period. Any person who died as the direct result of a fire, accidental or otherwise, was defined as a fire fatality in this study, except for those persons who died as a result of a fire associated with a motor vehicle accident. This category was excluded for three reasons: (1) these data were considerably more difficult to obtain; (2) it would be difficult to determine if the cause of death was the accident or the fire; (3) it was not felt that the inclusion of these fatalities would provide significant insight into the problems this study is addressing.

The fire fatalities were identified primarily through death certificates. In addition fire incident reports, fire casualty reports, state investigation reports, telephone inquiries, and personal contacts were used to identify additional fatalities, to identify non-fire fatalities which were incorrectly identified as fire fatalities on the death certificates, and to obtain the necessary information on the circumstances of each fatality. In each state the data collection effort was provided by or through the responsible state office with coordination and assistance from CFR as required.

For each state in the study the number of civilian residential fatalities identified and the time period used are shown in table 1 . In this report we will use the following terminology:

HIGH (LOW) refers to the set of data collected from the group of high (low) death rate states in this study.

ALL refers to the set of data collected from all of the states included in this study.

RURAL refers to the set of data collected from areas of < 2500 population. NON-RURAL refers to the set of data collected from areas of > 2500 population. Areas include municipalities, cities, towns and unincorporated areas.

Table 1. Civilian, residential fire death totals and fire death rates for HIGH and LOW states

\begin{tabular}{|c|c|c|c|c|}
\hline STATE & $\begin{array}{l}\text { NUMBER } \\
\text { OF DEATHS } \\
\end{array}$ & $\begin{array}{l}\text { POPULATION } \\
\text { (MILLION) }\end{array}$ & $\begin{array}{l}\text { FIRE DEATHS } \\
\text { PER MILLION }\end{array}$ & REPORTING PERIOD \\
\hline $\begin{array}{l}\text { AL } \\
\text { AR } \\
\text { GA } \\
\text { MS } \\
\text { OK } \\
\text { TN }\end{array}$ & $\begin{array}{r}163 \\
78 \\
227 \\
111 \\
89 \\
168 \\
\end{array}$ & $\begin{array}{l}3.742 \\
2.186 \\
5.084 \\
2.404 \\
2.880 \\
4.357 \\
\end{array}$ & $\begin{array}{l}43.6 \\
35.7 \\
44.6 \\
46.2 \\
30.9 \\
38.6 \\
\end{array}$ & $\begin{array}{l}\text { July } 1978 \text { - June } 1979 \\
\text { July } 1978 \text { - June } 1979 \\
\text { Jan. } 1978 \text { - Dec. } 1978 \\
\text { July } 1978 \text { - June } 1979 \\
\text { Jan. } 1978 \text { - Dec. } 1978 \\
\text { Jan. } 1978 \text { - Dec. } 1978\end{array}$ \\
\hline $\begin{array}{l}\text { HIGH } \\
\text { TOTAL }\end{array}$ & 836 & 20.652 & 40.5 & \\
\hline $\begin{array}{l}\text { CA } \\
C T \\
\text { DE } \\
\text { FI } \\
\text { UT } \\
\text { WI }\end{array}$ & $\begin{array}{r}355 \\
56 \\
27 \\
192 \\
23 \\
117 \\
\end{array}$ & $\begin{array}{r}22.294 \\
3.099 \\
0.583 \\
8.594 \\
1.307 \\
4.688 \\
\end{array}$ & $\begin{array}{l}15.9 \\
18.1 \\
46.3 \\
22.3 \\
17.6 \\
25.0 \\
\end{array}$ & $\begin{array}{l}\text { July } 1978 \text { - June } 1979 \\
\text { Jan. } 1978 \text { - Dec. } 1978 \\
\text { July } 1978 \text { - June } 1979 \\
\text { Jan. } 1978 \text { - Dec. } 1978 \\
\text { July } 1978 \text { - June } 1979 \\
\text { Jan. } 1978 \text { - Dec. } 1978\end{array}$ \\
\hline $\begin{array}{l}\text { LOW } \\
\text { TOTAL }\end{array}$ & 770 & 40.565 & 19.0 & \\
\hline ALL & 1606 & 61.217 & 26.2 & \\
\hline
\end{tabular}

2 Two different one-year periods were used, due to differences in reporting procedures among the states. 
Table 1 indicates that, state by state the death rate for the reporting period of this study in the HIGH states is considerably higher than the death rate in the LOW states, with the exception of Delaware. The choice of Delaware as a LOW state for this study was based on the USFA data available at that time [1], and Delaware's fatality rate had been low for several years. However, two fires resulting in thirteen fatalities during the study year increased Delaware's civilian residential fire-death rate to 46.3 per million. This type of result is not surprising for a state with both a small population and a generally low fire-death rate. Fatality rate calculations are generally done over a two or three year period to minimize these effects. When Delaware's fatality rate was calculated over several years, including the study period, it was concluded that Delaware should be included with the LOW states in this analysis.

Almost half of the LOW state fire fatalities occurred in California. It is possible, therefore, for trends that appear only in California to appear to be true for the entire group of LOW states. In order to guard against this possibility, various frequencies and data tabulations were compared for California versus the group of other LOW states. In most instances the Cailifornia data were similar to the data in the other states. There is considerable confidence, therefore, that the Low state results are not unduly influenced by California.

The overall civilian residential fire-death rate for the HIGH states is 40.5 deaths per million, more than double the overall 19.0 deaths per million in the LOW states.

In addition to the RURAL versus NON-RURAL population breakdowns, data were grouped and tabulated by 10 population intervals, as follows:

\section{Population Interval}

$\begin{array}{rrr}\text { 1. } & >1,000,000 \\ \text { 2. } & 500,000-1,000,000 \\ \text { 3. } & 250,000- & 500,000 \\ \text { 4. } & 100,000- & 250,000 \\ 5 . & 50,000- & 100,000 \\ 6 . & 25,000- & 50,000 \\ 7 . & 10,000- & 25,000 \\ \text { 8. } & 5,000- & 10,000 \\ 9 . & 2,500- & 5,000 \\ 10 . & <2,500\end{array}$

Each population interval includes the sum of the populations of all areas whose population falls within that interval for the particular set of areas (HIGH states, LOW states, etc.) under consideration.

All of the fire fatality data used in this study were obtained by the participating states and recorded on a worksheet. The data collected information about the individual fatality, such as age, sex, race, and place of residence. However, most of the data elements provide information about the fire. Most of these are based on the National Fire Protection Association (NFPA) 901, Uniform Coding for Fire Protection [3]. These data elements are familiar to the fire service community and provide a uniform way to consider fire "causes." A brief narrative of the fire was also included. Autopsy information was also requested when available. Finally, the sources for the data are indicated. The coding format used in this study is given in appendix $A$.

A worksheet was completed for every fire fatality identified. Thus if three persons died in a fire then three worksheets were completed. Once the fatalities were identified, an attempt was made to identify and contact the responding fire department. The contact was made by telephone, by mail, or by a personal visit. The completed worksheets were sent to the Center for Fire Research where they were reviewed, edited and coded for input to a computerized data base. The computer file was then edited, reviewed, and analyzed. 
Section 2 of this report discusses population estimates, including methodology and population distributions. Section 3 discusses overall death rates by population interval for HIGH, LOW, and ALL states. Section 4 discusses "causes" as defined by USFA [1], with emphasis on heating fire fatalities. (The methods used to define USFA "causes" are given in appendix A.) Section 5 describes the key fire scenarios identified as making up the bulk of the discrepancy between rural and non-rural fire fatality rates. Section 6 discusses general observations of interest in describing RURAL and NON-RURAL fatality rates, including race, condition of fatality at ignition, property type, detector presence, and fire department response. Section 7 provides an overview of the conclusions of the study.

The reader is cautioned that the data are not necessarily representative of all the fifty states as participating states were not selected according to a defined sampling plan. Also, since the methods used to identify the fatalities varied somewhat from state to state, the fire death rates for each state are approximate and individual states may not be directly comparable. In addition, uncertainties may be introduced by the method of calculating populations and by possible errors in the interpretation and coding of the worksheet information.

Additional data on fire fatalities are currently being collected, primarily from HIGH death rate states. A follow-up to this report will be issued when this expanded data base is available.

\section{2. 'POPULATION ESTIMATES}

The one objective of this study is to assess the differences between residential civilian fire fatalities in RURAL areas and fire fatalities in NON-RURAL areas. To do so it is first necessary to define the population of the area in which each fatality occurred.

The most appropriate means to compare RURAL and NON-RURAL fatalities was determined to be fatality rates, given here in fatalities per million persons. Using fatality rates as a measure eliminates the problems involving unequal population distributions which occur when using frequencies or percentages to compare characteristics of two or more distinct population groups. To use fatality rates, however, the total population in each population group under consideration (defined as Population Interval in Section 1) must be calculated.

This section discusses the approach used to define the population of the area in which each fatality occurred. Details of the methodology used and assumptions made in calculating total populations for each Population Interval are presented in appendix $B$.

\subsection{Population of Area where Fatality Occurred}

A section of the fire fatality worksheet asked the respondent to check off the appropriate population interval corresponding to the location where the fatal fire took place. While this provided a good first indication of populations, several problems were noted with these estimates. In some cases, populations were not known. In others, different respondents checked different population intervals for the same location. A more significant problem was the coding of the population interval corresponding to the location of the responding fire department rather than the location of the fatal fire. This sometimes resulted in RURAL areas being coded with the population interval corresponding to the population of an entire county when a county fire department responded. It also resulted in a rural or farm location, outside the city limits of a municipality, being coded with the population interval of that municipality.

To better define the actual populations of the fire location several steps were taken. Coded population intervals were compared with the populations given in the Rand McNally Commercial Atlas [4], and corrected where found to be incorrect. In some cases where the fire location was not adequately identified by place name, use of the zip code enabled identification of the place name. 
Finally, where death certificates defined the location of the fire as outside the city limits of small towns, or where addresses were obviously outside the limits of a populated area (such as "off Route 1" "7 miles outside town", "off Highway $355^{\circ}$ etc.) and where other factors such as run time, fire department identification, or descriptions given in the narrative indicated a rural location, the population interval was checked and corrected as necessary.

\subsection{Estimated Populations}

The estimated populations by population interval for HIGH and LOW states are given in tables 2 and 3 respectively.

Table 2. Population estimates HIGH rate states

\begin{tabular}{|c|c|c|c|c|c|c|c|}
\hline Population Interval & $\mathrm{AL}$ & $\overline{\mathrm{AR}}$ & $\overline{G A}$ & MS & $\overline{\mathrm{OK}}$ & TN & TOTAL \\
\hline$>1,000,000$ & - & - & - & - & - & - & \\
\hline $500,000-1,000,000$ & - & - & - & - & - & 674,269 & 674,269 \\
\hline $250,000-500,000$ & 325,000 & - & 455,300 & - & 745,000 & 471,006 & $1,996,306$ \\
\hline $100,000-250,000$ & 514,504 & 143,000 & 432,641 & 225,100 & - & 352,046 & $1,667,291$ \\
\hline $50,000-100,000$ & 72,400 & $190,654^{\circ}$ & 175,681 & - & 258,540 & 55,323 & 752,598 \\
\hline $25,000-$ & 444,672 & 212,149 & 388,259 & 389,801 & 277,624 & 304,740 & $2,017,245$ \\
\hline $10,000-$ & 443,396 & 354,377 & 855,441 & 371,613 & 424,931 & 504,694 & $2,954,452$ \\
\hline 10,000 & 326,614 & 204,703 & 463,417 & 164,012 & 220,373 & 259,021 & $1,638,140$ \\
\hline 2,500 & 227,378 & 160,474 & 415,728 & 132,475 & 186,102 & 184,862 & $1,307,019$ \\
\hline$<2500$ & $1,387,942$ & 920,616 & $1,897,405$ & $1,120,901$ & 767,243 & $1,550,831$ & $7,644,938$ \\
\hline TOTAL & $3,741,906$ & $2,185,973$ & $5,083,872$ & $2,403,902$ & $2,879,812$ & $4,356,793$ & $20,652,258$ \\
\hline
\end{tabular}

Table 3. Population estimates LOW rate states

\begin{tabular}{|c|c|c|c|c|c|c|c|}
\hline Population Interval & $\overline{C A}$ & $\mathrm{CT}$ & $\overline{D E}$ & $\overline{F L}$ & UT & WI & TOTAL \\
\hline$>1,000,000$ & $2,900,838$ & - & - & - & - & - & $2,900,838$ \\
\hline $500,000-1,000,000$ & $2,068,500$ & - & - & 584,252 & - & 613,190 & $3,265,942$ \\
\hline $250,000-500,000$ & 945,900 & - & - & 645,300 & - & - & $1,591,200$ \\
\hline $100,000-250,000$ & $2,213,731$ & 633,947 & - & 895,959 & 180,000 & 173,051 & $4,096,688$ \\
\hline $50,000-100,000$ & $3,950,174$ & 765,505 & 169,286 & 443,251 & 143,393 & 594,314 & $6,065,923$ \\
\hline $25,000-$ & $5,151,190$ & 627,671 & 137,695 & $2,003,628$ & 214,380 & 446,103 & $8,580,667$ \\
\hline $10,000-$ & $2,753,791$ & 622,953 & 62,242 & $1,364,869$ & 258,242 & 579,909 & $5,642,006$ \\
\hline 10,000 & 819,625 & 232,839 & 75,869 & 761,757 & 188,707 & 449,716 & $2,528,513$ \\
\hline $2,500-$ & 418,062 & 147,940 & 15,173 & 453,159 & 126,135 & 490,968 & $1,651,437$ \\
\hline$<2500$ & $1,072,294$ & 68,073 & 122,768 & $1,441,662$ & 196,086 & $1,340,987$ & $4,241,870$ \\
\hline TOTAL & $22,294,105$ & $3,098,928^{\circ}$ & 583,033 & $8,593,837$ & $1,306,943$ & $4,688,238$ & $40,565,084$ \\
\hline
\end{tabular}




\section{OVERALI FATALITY RATES}

The populations by population interval described in Section 2 were matched with the civilian residential fatalities in each population interval and fatality rates by population interval for HIGH, LOW and ALL states were calculated. The results are presented in tables 4,5 , and 6 respectively, as fatality rates per million population. The overall residential civilian fatality rate in this study of about 26 per million is slightly lower than national estimates of about 30 per million. but the difference is not believed to be significant. The total death rate for all fires in this study (residential and non-residential) was about 29 per million, also slightly lower than the national average (excluding transportation) of about 32 per million.

Table 4. Fatality rates by population interval - HIGH rate states

\begin{tabular}{|c|c|c|c|c|}
\hline Population & Interval & $\begin{array}{l}\text { Estimated } \\
\text { Population }\end{array}$ & $\begin{array}{l}\text { Civilian } \\
\text { Deaths }\end{array}$ & $\begin{array}{c}\text { Death } \\
\text { Rate/Million }\end{array}$ \\
\hline$>1,000$ & 0,000 & $\mathrm{~N} / \mathrm{A}$ & $N / A$ & $\mathrm{~N} / \mathrm{A}$ \\
\hline 2. 500,000 & $=1,000,000$ & 674,269 & 18 & 26.7 \\
\hline 3. $250,000-$ & $-\quad 500,000$ & $1,996,306$ & 101 & 50.6 \\
\hline 4. $100,000-$ & 250,000 & $1,667,291$ & 61 & 36.6 \\
\hline $50,000-$ & 100,000 & 752,598 & 31 & 41.2 \\
\hline $25,000-$ & 50,000 & $2,017,245$ & 51 & 25.3 \\
\hline $10,000-$ & 25,000 & $2,954,452$ & 68 & 23.0 \\
\hline $5,000 \ldots$ & 10,000 & $1,638,140$ & 43 & 26.2 \\
\hline $2,500-$ & 5,000 & $1,307,019$ & 42 & 32.1 \\
\hline$<2,500$ & & $7,644,938$ & $\underline{421}$ & 55.1: \\
\hline & TOTAL & $20,652,258$ & 836 & 40.5 \\
\hline
\end{tabular}

Table 5. Fatality rates by population interval - Low rate states

\begin{tabular}{|c|c|c|c|c|c|}
\hline \multicolumn{3}{|c|}{ Population Interval } & \multirow{2}{*}{$\begin{array}{l}\begin{array}{l}\text { Estimated } \\
\text { Population }\end{array} \\
2,900,838\end{array}$} & $\begin{array}{l}\text { Civilian } \\
\text { Deaths }\end{array}$ & \multirow{2}{*}{$\begin{array}{c}\text { Death } \\
\text { Rate/Million }\end{array}$} \\
\hline 1. & $>1,000$ & 0,000 & & 68 & \\
\hline 2 . & 500,000 & $-1,000,000$ & $3,265,942$ & 69 & 21.1 \\
\hline 3. & 250,000 & $-\quad 500,000$ & $1,591,200$ & 54 & 33.9 \\
\hline 4 . & 100,000 & 250.000 & $4,096,688$ & 82 & 20.0 \\
\hline 5 . & 50,000 & 100,000 & $6,065,923$ & 104 & 17.1 \\
\hline 6. & 25,000 & 50,000 & $8,580,667$ & 75 & 8.7 \\
\hline 7. & 10,000 & 25,000 & $5,642,006$ & 72 & 12.8 \\
\hline 8 . & 5,000 & 10,000 & $2,528,513$ & 33 & 13.1 \\
\hline 9. & 2,500 & 5,000 & $1,651,437$ & 26 & 15.7 \\
\hline \multirow[t]{2}{*}{10.} & $<2,50$ & 0 & $4,241,870$ & 181 & $\underline{42.7}$ \\
\hline & & TOTAL & $\overline{40,565,084}$ & $764^{3}$ & 18.8 \\
\hline
\end{tabular}

\footnotetext{
The population interval was not known for 6 fatalities.
} 
Table 6. Fatality rates by population interval - ALL states

\begin{tabular}{|c|c|c|c|c|c|}
\hline \multicolumn{3}{|c|}{ Population Interval } & $\begin{array}{l}\text { Estimated } \\
\text { Population }\end{array}$ & $\begin{array}{l}\text { Civilian } \\
\text { Deaths }\end{array}$ & $\begin{array}{c}\text { Death } \\
\text { Rate/Million }\end{array}$ \\
\hline \multicolumn{3}{|c|}{$>1,000,000$} & $2,900,838$ & 68 & \multirow{2}{*}{$\begin{array}{l}23.4 \\
22.1\end{array}$} \\
\hline 2. & & $3,940,211$ & 87 & \\
\hline 3. & & & $3,587,506$ & 155 & 43.2 \\
\hline 4. & \multicolumn{2}{|c|}{$100,000-250,000$} & $5,763,979$ & 143 & 24.8 \\
\hline 5. & \multicolumn{2}{|c|}{$50,000-100,000$} & $6,818,521$ & 135 & 19.8 \\
\hline 6. & \multicolumn{2}{|c|}{$25,000-50,000$} & $10,597,911$ & 126 & 11.9 \\
\hline 7. & $10,000-$ & 25,000 & $8,596,458$ & 140 & 16.3 \\
\hline 8. & $5,000-$ & 10,000 & $4,166,653$ & 76 & 18.2 \\
\hline 9 . & $2,500-$ & 5,000 & $2,958,456$ & 68 & 23.0 \\
\hline \multirow[t]{2}{*}{10.} & \multicolumn{2}{|l|}{$<2,500$} & $11,886,808$ & 602 & 50.6 \\
\hline & \multicolumn{2}{|r|}{ TOTAL } & $61,217,341$ & 1600 & 26.1 \\
\hline
\end{tabular}

As these tables demonstrate, the RURAL (< 2500 population) fire fatality rate is considerably higher than the rates for the other population intervals. The HIGH states also have a significantly higher fatality rate than the LOW states. Figure 1 , uses linear graphs of fatality rates by population interval to more clearly depict the pattern of fatality rates by population interval.

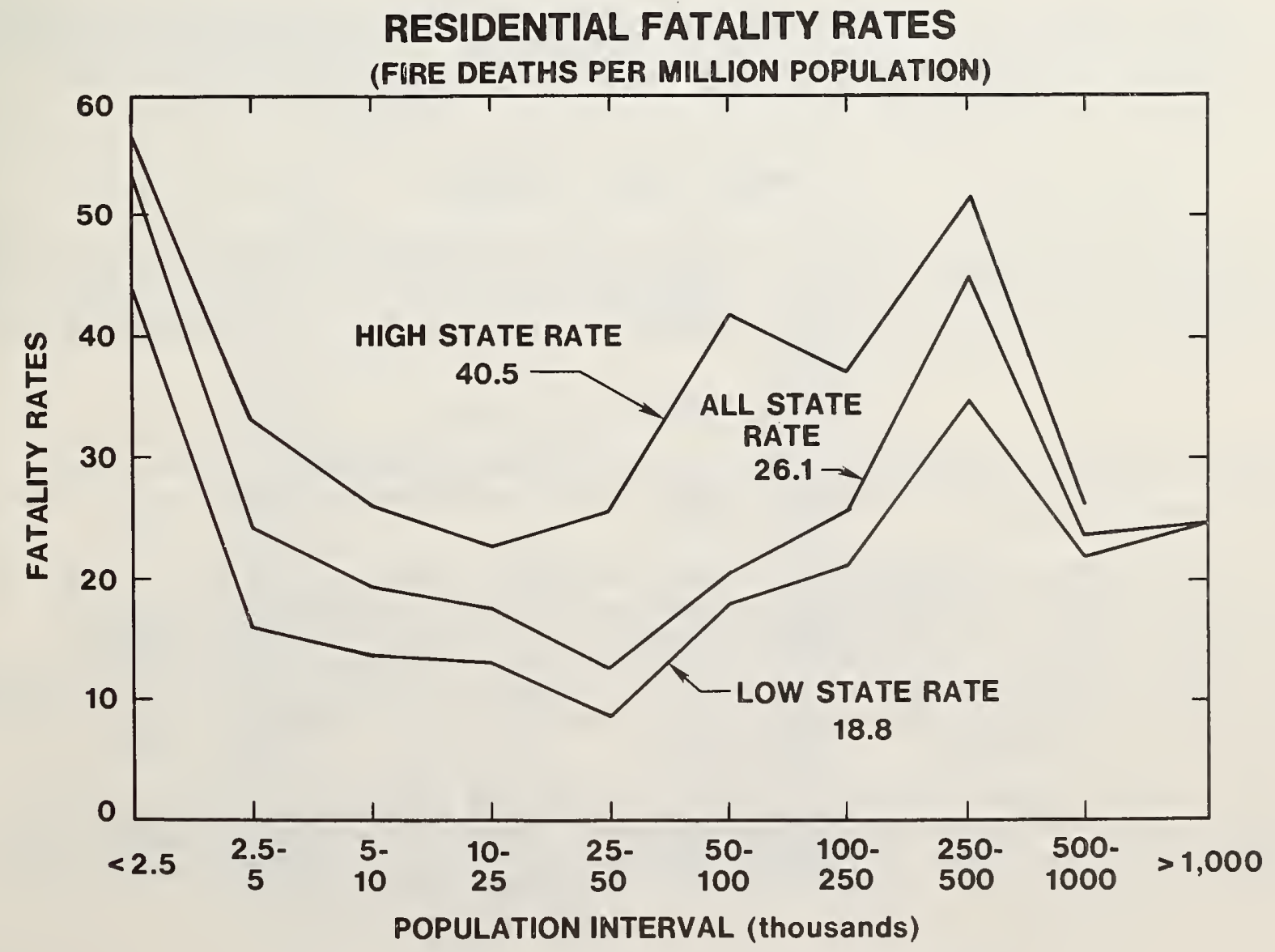

Figure 1. Residential fatality rates - All states 
The characteristic "bathtub" curve previously noted by other sources, including NFPA [5] and USFA [2] is evident in this data base also.

Since the purpose of this study is to compare RURAI and NON-RURAI fatality rates, and since the number of fatalities in individual population intervals over 2500 was relatively small, most analysis was done using only RURAL $(<2500)$ and NONRURAL (> 2500) comparisons. In several cases, however, the use of general categories of comparison, such as USFA "cause," enabled us to compare and contrast rates by individual population interval.

Also of significance is that the HIGH and LOW states produced similarly shaped curves, indicating that a rural fire problem exists regardless of overall death rate. states.

Table 7 compares RURAL and NON-RURAL fatality rates for HIGH, LOW, and ALL

Table 7. RURAL and NON-RURAL fatality rates

RURAL HIGH NON-RURAL RURAL ${ }^{\text {LOW NON-RURAL RURAL }}$ ALL NON-RURAL
55.1
31.9
42.7
16.1
50.6
20.2

Overall, the RURAL fatality rate is 2.5 times the NON-RURAL rate. Further details of the causal factors contributing to the RURAL and NON-RURAL fatality rates in both HIGH and LOW states are presented in section 5 .

It should also be noted that the contribution of RURAL fatalities in the HIGH states to the HIGH state fatality rates is considerably greater than the contribution of RURAL fatalities in the LOW states, since a greater proportion of the population in the HIGH states is rural.

\section{GENERAL CAUSAL PATTERNS}

The objective of this study is to assess the differences between RURAL and NONRURAL fatalities and to determine if causal factors responsible for the higher RURAI fire fatality rate can be identified. This initial analysis of causal patterns uses the USFA developed "causes" (appendix A) which categorize the basic causal factors . of fires into a set of general causes.

\subsection{Fatality Rates by Cause}

Table 8 gives the fatality rate by cause for each population interval. As indicated in table 8 , there are significant differences between RURAL and NON-RURAI areas in terms of cause.

The largest RURAL/NON-RURAL difference is unknown cause. This may be due, in part, to longer RURAL response times and delayed alarms, resulting in greater physical destruction and a consequent increase in the difficulty of successful investigation. Another factor may be a lack of resources for investigation in RURAL areas. Other factors may be at work as well. Since it is not possible to investigate unknown causes in further detail they are excluded from the remaining analyses which. concentrate on known causes.

of the known causes, heating is shown to have the largest discrepancy between RURAL (13.8) and NON-RURAL (3.3) fatality rates. Cooking also has a considerably higher RURAL fatality rate, although it should also be noted that all causes (except incendiary/suspicious) had higher RURAL than NON-RURAI fatality rates. 
Table 8. Fatality rates by cause

\begin{tabular}{|c|c|c|c|c|c|c|c|c|c|c|c|c|}
\hline \multirow[b]{2}{*}{ Cause } & \multirow{2}{*}{$\begin{array}{l}\text { Rural } \\
\text { Rate } \\
<2.5\end{array}$} & \multicolumn{9}{|c|}{ Population Interval $\left(1000^{\prime} \mathrm{s}\right)$} & \multirow{2}{*}{$\begin{array}{l}\text { Non- } \\
\text { Rural } \\
\text { Rate }\end{array}$} & \multirow{2}{*}{$\begin{array}{c}\text { Over- } \\
\text { all } \\
\text { Rate }\end{array}$} \\
\hline & & $2 \cdot 5-5$ & $5-10$ & $10-25$ & $25-50$ & $50-100$ & $100-250$ & $250-500$ & $500-1 \mathrm{~m}$ & $>1 \mathrm{~m}$ & & \\
\hline Heating & 13.8 & 4.4 & 4.1 & 3.3 & 2.1 & 2.8 & 3.0 & 7.0 & 4.1 & 1.7 & 3.3 & 5.3 \\
\hline Cooking & 4.2 & 2.0 & 2.6 & 2.0 & 0.7 & 0.7 & 1.2 & 2.0 & 1.3 & 1.0 & 1.4 & 1.9 \\
\hline $\begin{array}{r}\text { Incendiary/ } \\
\text { Suspicious }\end{array}$ & 0.7 & 1.0 & 1.2 & 1.5 & 0.9 & 2.5 & 1.7 & 4.7 & 2.0 & 4.5 & 2.0 & 1.7 \\
\hline Smoking & 7.3 & 4.7 & 3.8 & 3.8 & 2.9 & 6.5 & 8.5 & 13.7 & 5.3 & 11.0 & 5.9 & 6.1 \\
\hline Elec. Dist. & 2.5 & 3.4 & 0.5 & 1.1 & 1.3 & 0.3 & 1.9 & 1.4 & 0.8 & - & 1.1 & 1.4 \\
\hline Appliances & 1.2 & 0.7 & 1.0 & 0.1 & 0.7 & 0.7 & 0.5 & 1.4 & - & 0.3 & 0.6 & 0.7 \\
\hline $\begin{array}{c}\text { Children } \\
\text { Playing }\end{array}$ & 1.1 & 0.7 & 1.4 & 0.1 & 0.6 & 0.9 & 0.5 & 3.9 & 1.8 & 0.7 & 1.0 & 1.0 \\
\hline Open Flame & 1.6 & 1.7 & 0.2 & 0.6 & 0.6 & 0.9 & 1.4 & 1.4 & 1.8 & 1.4 & 1.0 & 1.1 \\
\hline other & 1.0 & - & - & - & - & 0.2 & - & 0.8 & - & 0.3 & 0.1 & 0.3 \\
\hline Unknown & 17.2 & 4.4 & $3: 4$ & 3.8 & 2.2 & 4.4 & 6.1 & 7.0 & 5.1 & 2.4 & 4.1 & 6.6 \\
\hline TOTAL & 50.6 & 23.0 & 18.2 & 16.3 & 12.0 & 19.9 & 24.8 & 43.3 & 22.2 & 23.3 & 20.5 & 26.1 \\
\hline
\end{tabular}

NOTE: Totals may not agree with table 6 due to rounding errors.

Fatality rates are given in fire deaths per million population.

The patterns of fatality rate by cause for each population interval for ALL states are illustrated in figure 2. Disregarding unknown, heating dominates only in the < 2500 population interval. The contribution of smoking as a cause appears to increase as population increases, as does that of incendiary/suspicious. The contribution of cooking and heating appear to decrease with increasing population. Related patterns are illustrated in figure 3, which shows fatality rates by population interval for each cause. Figure 4 compares RURAL and NON-RURAL fatality rates for the significant "causes" in the HIGH states with those in the LOW states.

\section{FATALITY RATES BY CAUSE FOR EACH POPULATION INTERVAL}
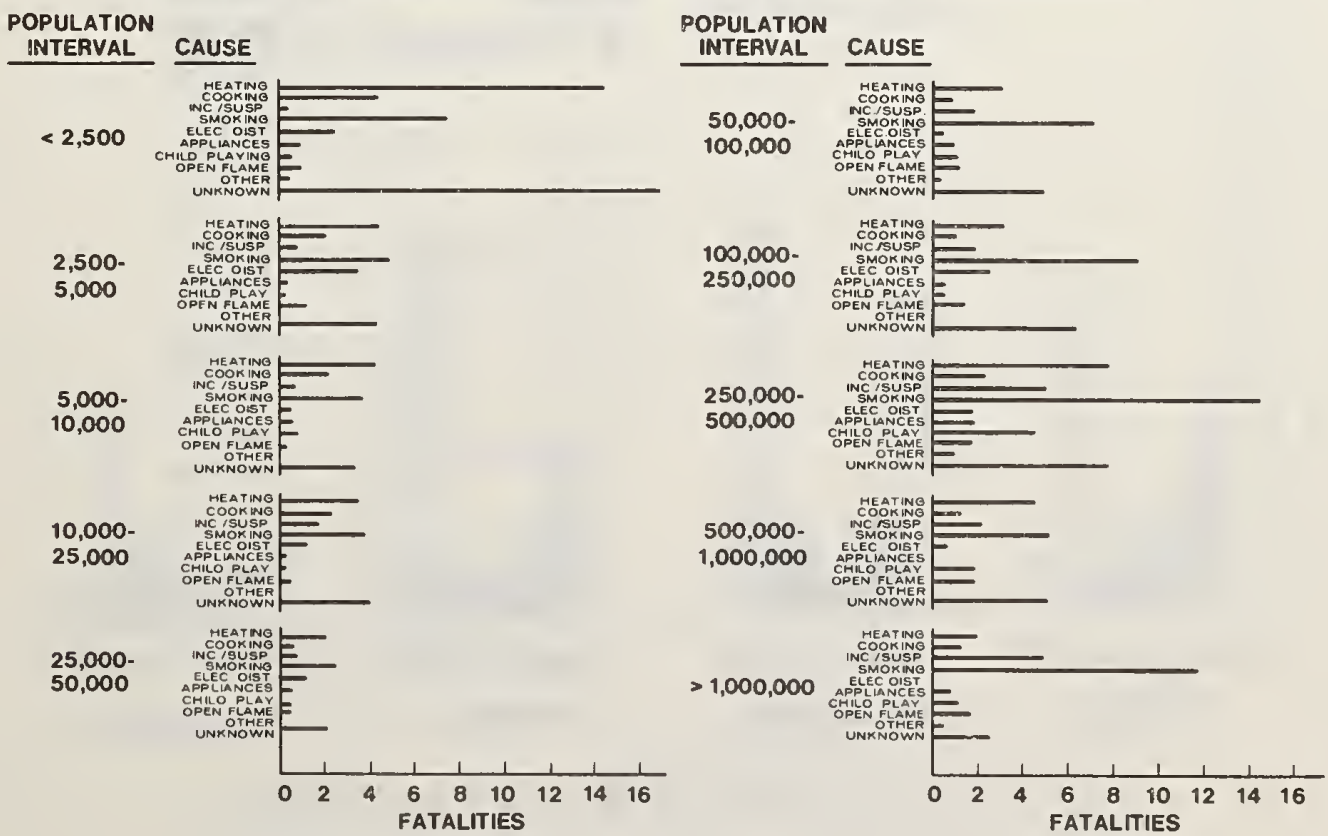

Figure 2. FAtALITY RATES by CAUSE FOR POPULATION INTERVALS 
FATALITY RATES BY POPULATION INTERVAL FOR EACH CAUSE

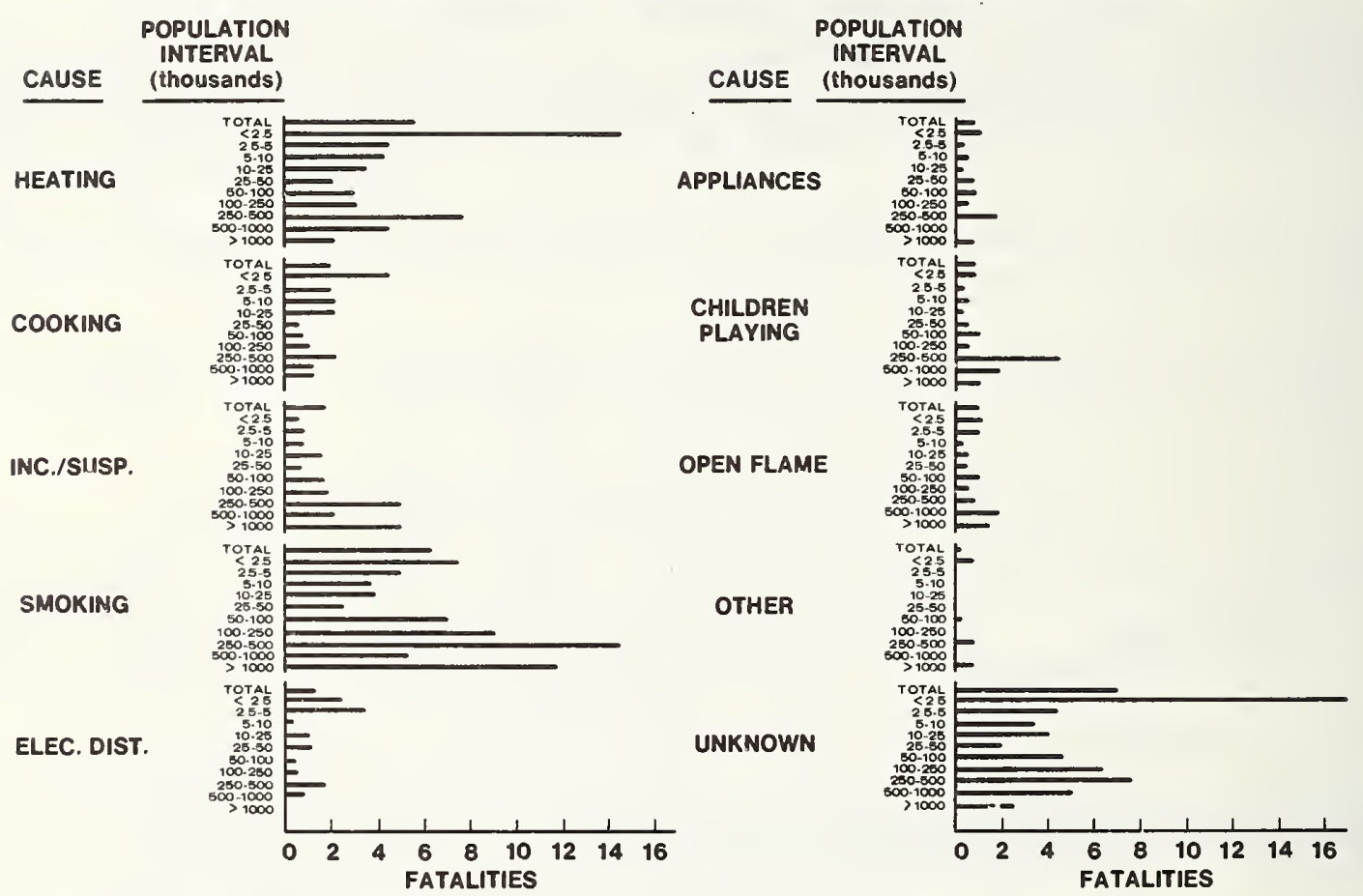

Figure 3. Fatality Rates by POPUlation Interval For EACH CAUSE

FATALITY RATES BY CAUSE - HIGH AND LOW STATES

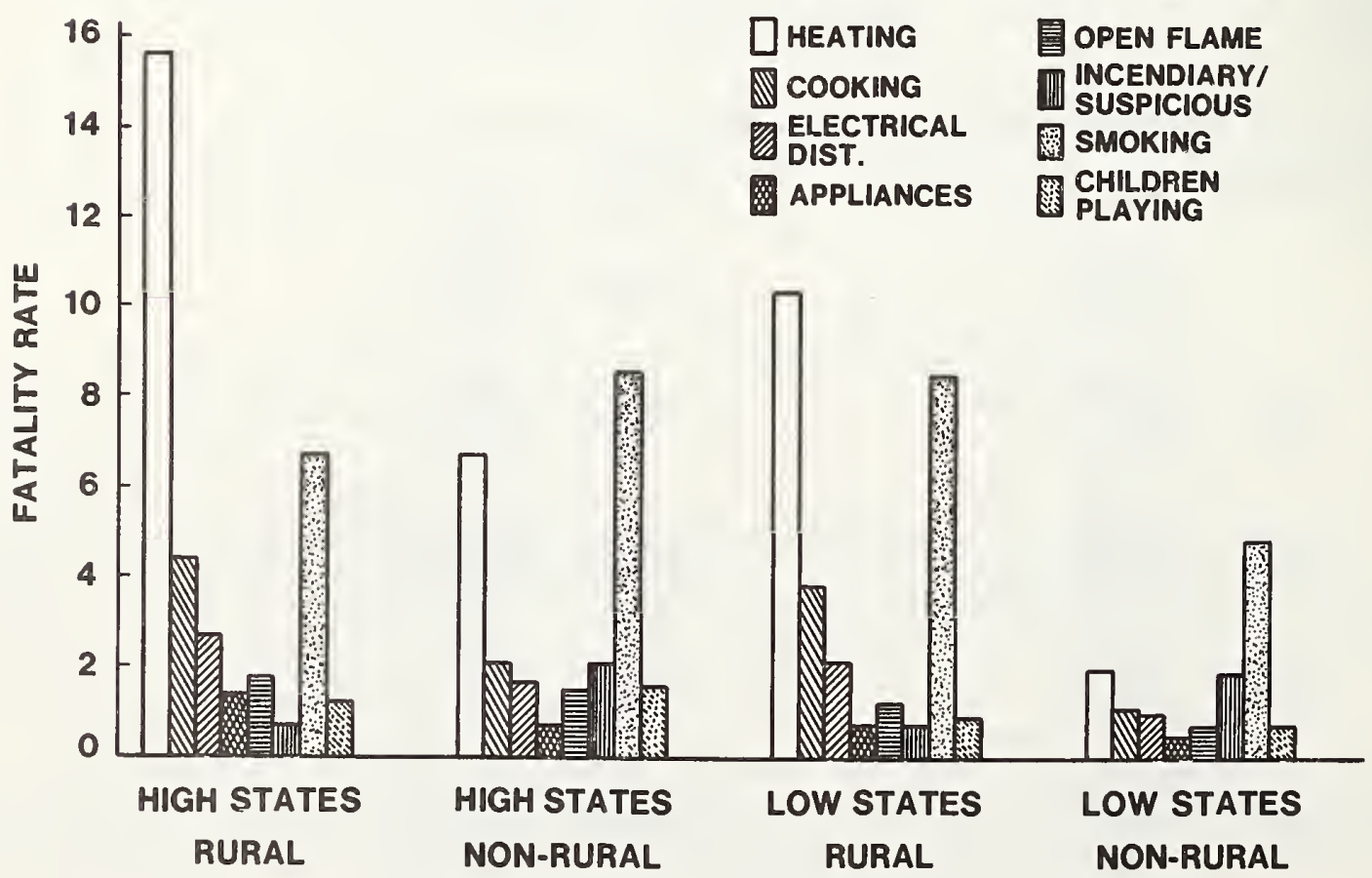

Figure 4. Fatality Rates by cause for hIGH and LOW states 
A similar pattern is present for the heating, cooking and electrical distribution causes. For these causes, the highest fatality rates are recorded for the HIGH RURAL category, followed by the LOW RURAL, the HIGH NON-RURAL and finally the LOW NON-RURAL. (Unknown fires also exhibit this pattern.) Similar, but less distinct, patterns also occur for appliance and open flame causes. Heating exhibits the most pronounced pattern, as well as having the highest fatality rates. These distributions indicate that for most fire causes a pattern of higher RURAL than NON-RURAL fatality rates exists for both HIGH and LOW states, with the RURAL/NON-RURAL fatality rate discrepancy being more severe than the HIGH/LOW discrepancy.

Conversely, the NON-RURAL fatality rates predominate in the case of the incendiary/suspicious cause, and the $\mathrm{HIGH}$ state fatality rates predominate slightly in the smoking and children playing causes.

\subsection{Type of Fuel in Heating Fires}

Since heating is the cause with the most significant RURAL/NON-RURAL fatality rate difference an additional detail, type of fuel, was examined for the heating cause. The results (with unknown cause redistributed in accordance with the distribution of the "known's") are illustrated in figure 5. It is apparent that the RURAL heating fire fatality problem is predominately solid fueled heating equipment.

of the solid fueled heating equipment fire fatalities, 55 percent were in HIGH RURAI areas, 21 percent in LOW RURAL areas, 20 percent in HIGH NON-RURAL areas, and 4 percent in LOW NON-RURAL areas.

\section{COMPARISON OF FATALITIES BY HEATING EQUIPMENT AND FUEL}

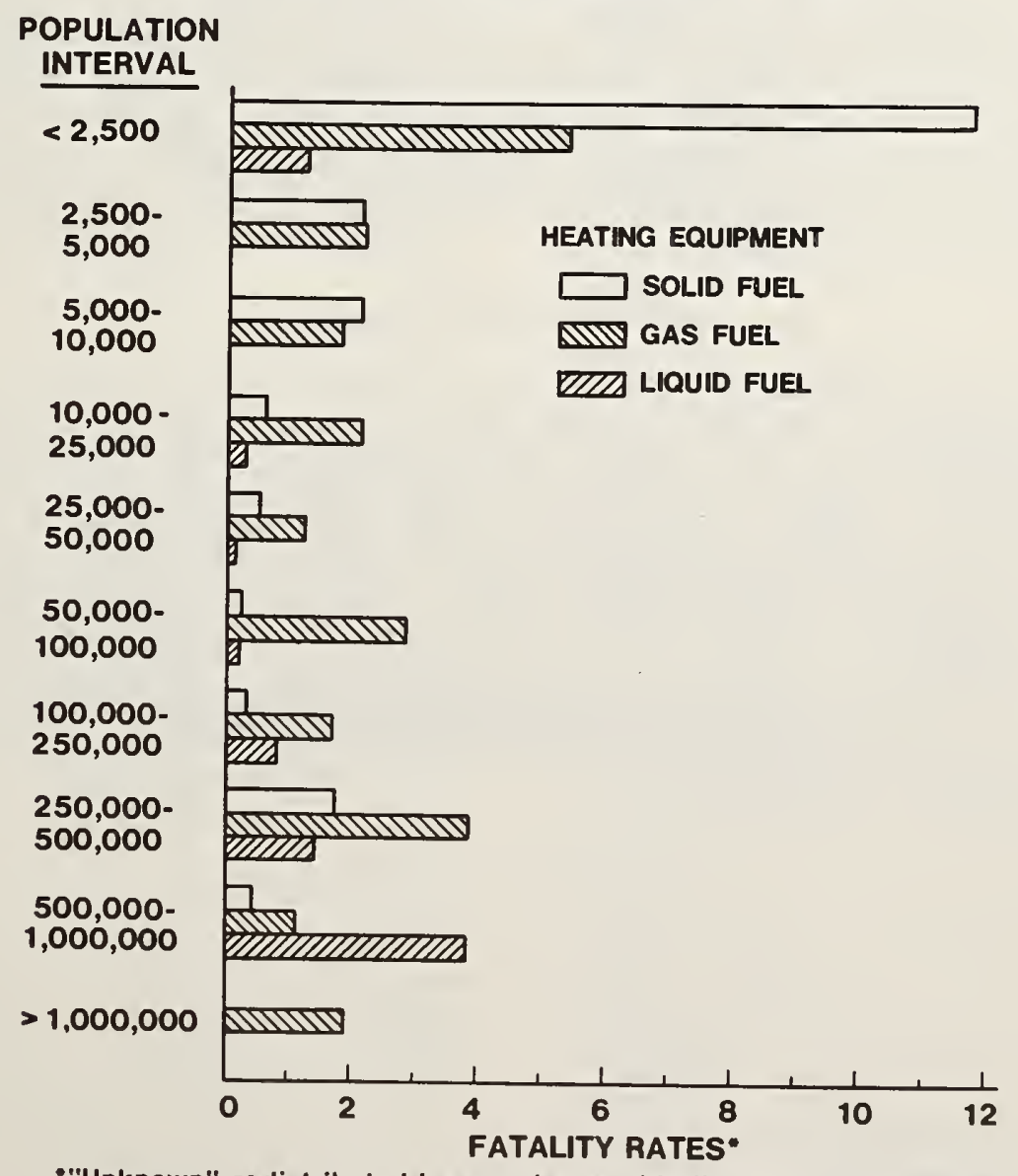

" Unknown" redistributed in accordance with distribution of "Known"

Figure 5. Fatalities by heating equipment and fuel 
While these general discussions of causes provide an overview of the problem, they are not specific enough to describe the differences in RURAI and NON-RURAI fatality rates in detail or to direct action. The next section breaks down the RURAL/NON-RURAL differences into greater detail, using descriptive scenarios to illustrate the nature of the differences, and delves further into the RURAI/NONRURAL differences in the HIGH and LOW states.

\section{FIRE FATALITY SCENARIOS}

In order to identify more specifically the characteristics of RURAL fires which contribute most to the high RURAL fire rate a series of fire scenarios were identified by combining relevant causal factors and comparing the resultant RURAL and NON-RURAI fatality rates for ALL states. The great majority of the identifiable RURAL/NONRURAL discrepancy in fire fatality rates for ALI states is due to fires with the characteristics described in these scenarios.

The scenario descriptions in this section do not specifically describe all fires in a given scenario, but provide a general description of the most common types of fires which fall within a scenario. The scenario descriptions are followed by a brief summary statement which describes the key factors (Equipment Involved in Ignition, Form of Heat of Ignition, etc) in the scenario. Also included are tabulations of the fatality rates by these factors, for ALL states and for HIGH and LOW states separately. Where appropriate the differences in the RURAL/NON-RURAL fire fatality characteristics between the HIGH and LOW states are also discussed.

These scenarios provide an initial description of some of the significant characteristics of the RURAL fire problem. They should not be considered final and definitive, particularly in view of the uncertainties in calculations of populations described previously, and the large number of unknown RURAL fire fatalities. There may also be elements involving areas such as income level or housing stock characteristics which relate to, or are common to with, many scenarios. The characteristics described in these scenarios do, however, provide an initial indication of possible approaches toward addressing the RURAL fire problem.

\subsection{Heating Fire Scenarios}

The fatality rate in RURAL heating fires ${ }^{4}$ (13.8) is over four times the fatality rate in NON-RURAL heating fires (3.3). Tables 9-12 show the fatality rates in RURAL and NON-RURAL heating fires for ALI states by Equipment Involved in Ignition (EQP) and Form of heat of Ignition (FHI), for the four significant categories of Form of Material Ignited (FMI). These categories are, respectively, structural (walls, framing, etc.), furnishings and miscellaneous (bedding, trash, etc.), clothing on person, and flammable liquids and gases. The major differences between the RURAI, and NON-RURAL heating fire fatality rates are drawn from tables $9-12$ and are described by six scenarios. These scenarios describe the types of RURAL heating fires which produce most of this discrepancy between RURAL and NON-RURAL heating fire fatality rates. Tables 13-16 further differentiate RURAL and NON-RURAL heating fire scenarios by HIGH and LOW states.

${ }^{4}$ The fatality rate in the RURAL population due to heating fires. 
Table 9. Heating fire fatality rates - ALL states

(Form of material ignited: walls, ceiling, framing, insulation, flooring)

\begin{tabular}{|c|c|c|c|c|c|}
\hline EQP FHI & $\begin{array}{l}\text { GAS } \\
\text { FUEL }\end{array}$ & $\begin{array}{c}\text { LIQUID } \\
\text { FUEL }\end{array}$ & $\begin{array}{l}\text { SOLID } \\
\text { FUEL }\end{array}$ & $\begin{array}{l}\text { ELEC- } \\
\text { TRIC }\end{array}$ & OTHER \\
\hline $\begin{array}{l}\text { Central } \\
\text { Heating }\end{array}$ & 0.34 & & & & \\
\hline $\begin{array}{l}\text { Water } \\
\text { Heater }\end{array}$ & 0.34 & & & & \\
\hline $\begin{array}{l}\text { Local } \\
\text { Heater }\end{array}$ & 0.08 & & 1.51 & 0.08 & \\
\hline Fireplace & & & 0.67 & & \\
\hline $\begin{array}{l}\text { Portable } \\
\text { Heater }\end{array}$ & & 0.42 & 0.50 & 0.08 & \\
\hline Chimney & & & 0.25 & & \\
\hline
\end{tabular}

ALL RURAI

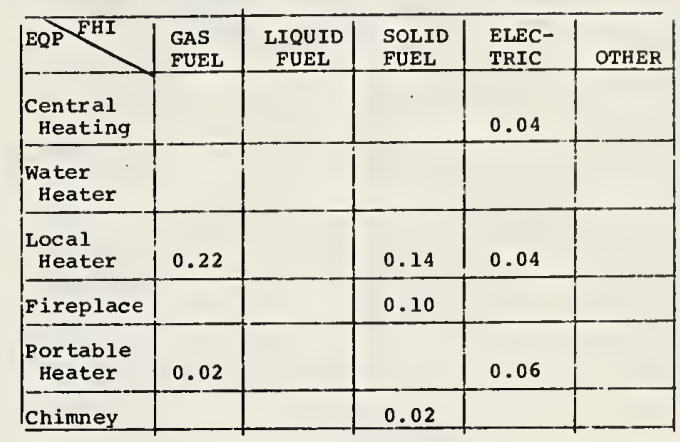

ALL NON-RURAL

Table 10. Heating fire fatality rates - ALL states

(Form of material ignited: furniture, bedding, soft goods, trash)

\begin{tabular}{|c|c|c|c|c|c|}
\hline EQP FHI & $\begin{array}{l}\text { GAS } \\
\text { FUEL }\end{array}$ & $\begin{array}{l}\text { LIQUID } \\
\text { FUEL }\end{array}$ & $\begin{array}{l}\text { SOLID } \\
\text { FUEL }\end{array}$ & $\begin{array}{l}\text { ELEC- } \\
\text { TRIC }\end{array}$ & OTHER \\
\hline \multicolumn{6}{|l|}{$\begin{array}{l}\text { Central } \\
\text { Heating }\end{array}$} \\
\hline \multicolumn{6}{|l|}{$\begin{array}{l}\text { Water } \\
\text { Heater }\end{array}$} \\
\hline $\begin{array}{l}\text { Local } \\
\text { Heater }\end{array}$ & 0.25 & & 0.17 & 0.34 & \\
\hline Fireplace & & & 0.59 & & \\
\hline $\begin{array}{l}\text { Portable } \\
\text { Heater }\end{array}$ & 0.08 & 0.08 & & 0.50 & \\
\hline Chimney & & & & & \\
\hline
\end{tabular}

ALL RURAL

\begin{tabular}{|l|l|l|l|l|l|}
\hline EQP FHI & $\begin{array}{l}\text { GAS } \\
\text { FUEL }\end{array}$ & $\begin{array}{c}\text { LIQUID } \\
\text { FUEL }\end{array}$ & $\begin{array}{l}\text { SOLID } \\
\text { FUEL }\end{array}$ & $\begin{array}{l}\text { ELEC- } \\
\text { TRIC }\end{array}$ & OTHER \\
\hline $\begin{array}{l}\text { Central } \\
\text { Heating }\end{array}$ & & & & & \\
\hline $\begin{array}{l}\text { Water } \\
\text { Heater }\end{array}$ & 0.02 & & 0.06 & & \\
\hline $\begin{array}{l}\text { Local } \\
\text { Heater }\end{array}$ & 0.25 & & 0.04 & & \\
\hline $\begin{array}{l}\text { Fireplace } \\
\text { Portable } \\
\text { Heater }\end{array}$ & 0.02 & 0.02 & & 0.36 & \\
\hline Chimney & & & & & \\
\hline
\end{tabular}

ALI NON-RURAL

Table 11. Heating fire fatality rates - ALL states

(Form of material ignited: garment on person)

\begin{tabular}{|l|l|l|l|l|l|}
\hline EQP FHI & $\begin{array}{c}\text { GAS } \\
\text { FUEL }\end{array}$ & $\begin{array}{c}\text { LIQUID } \\
\text { FUEL }\end{array}$ & $\begin{array}{c}\text { SOLID } \\
\text { FUEL }\end{array}$ & $\begin{array}{l}\text { ELEC- } \\
\text { TRIC }\end{array}$ & OTHER \\
\hline $\begin{array}{l}\text { Central } \\
\text { Heating }\end{array}$ & & & & & \\
\hline $\begin{array}{l}\text { Water } \\
\text { Heater }\end{array}$ & & & 0.25 & & 0.08 \\
\hline $\begin{array}{l}\text { Local } \\
\text { Heater }\end{array}$ & 0.67 & & 1.01 & & \\
\hline $\begin{array}{l}\text { Fireplace } \\
\text { Portable } \\
\text { Heater }\end{array}$ & & & & 0.17 & 0.08 \\
\hline Chimney & & & & & \\
\hline
\end{tabular}

ALL RURAI

\begin{tabular}{|c|c|c|c|c|c|}
\hline EQP FHI & $\begin{array}{l}\text { GAS } \\
\text { FUEL }\end{array}$ & $\begin{array}{c}\text { LIQUID } \\
\text { FUEL }\end{array}$ & $\begin{array}{c}\text { SOLID } \\
\text { FUEL }\end{array}$ & $\begin{array}{c}\text { ELEC- } \\
\text { TRIC }\end{array}$ & OTHER \\
\hline $\begin{array}{c}\text { Central } \\
\text { Heating }\end{array}$ & & & & & \\
\hline $\begin{array}{c}\text { Water } \\
\text { Heater }\end{array}$ & & 0.02 & 0.02 & \\
\hline $\begin{array}{c}\text { Local } \\
\text { Heater }\end{array}$ & 0.20 & 0.04 & & \\
\hline Fireplace & & 0.06 & 0.02 & \\
\hline $\begin{array}{c}\text { Portable } \\
\text { Heater }\end{array}$ & 0.02 & & & & \\
\hline Chimney & & & & & \\
\hline
\end{tabular}

ALL NON-RURAL

Table 12. Heating fire fatality rates - ALL states (Form of material ignited: flammable liquid, fuel)

\begin{tabular}{|c|c|c|c|c|c|}
\hline EQP FHI & $\begin{array}{l}\text { GAS } \\
\text { FUEL } \\
\end{array}$ & $\begin{array}{l}\text { LIQUID } \\
\text { FUEL } \\
\end{array}$ & $\begin{array}{l}\text { SOLID } \\
\text { FUEL }\end{array}$ & $\begin{array}{l}\text { ELEC- } \\
\text { TRIC }\end{array}$ & OTHER \\
\hline \multicolumn{6}{|l|}{$\begin{array}{l}\text { Central } \\
\text { Heating }\end{array}$} \\
\hline $\begin{array}{l}\text { Water } \\
\text { Heater }\end{array}$ & 0.50 & & & & \\
\hline $\begin{array}{l}\text { Local } \\
\text { Heater }\end{array}$ & 0.08 & & 0.42 & & 0.17 \\
\hline Fireplace & & & 0.08 & & 0.08 \\
\hline $\begin{array}{l}\text { Portable } \\
\text { Heater }\end{array}$ & 0.08 & 0.08 & & & \\
\hline Chimney & & & & & \\
\hline
\end{tabular}

ALL RURAL

\begin{tabular}{|c|c|c|c|c|c|}
\hline EQP FHI & $\begin{array}{c}\text { GAS } \\
\text { FUEL }\end{array}$ & $\begin{array}{c}\text { LIQUID } \\
\text { FUEL }\end{array}$ & $\begin{array}{c}\text { SOLID } \\
\text { FUEL }\end{array}$ & $\begin{array}{c}\text { ELEC- } \\
\text { TRIC }\end{array}$ & OTHER \\
\hline $\begin{array}{c}\text { Central } \\
\text { Heating }\end{array}$ & & & & & \\
\hline $\begin{array}{c}\text { Water } \\
\text { Heater }\end{array}$ & 0.32 & 0.04 & 0.02 & & 0.02 \\
\hline $\begin{array}{c}\text { Local } \\
\text { Heater }\end{array}$ & 0.14 & 0.28 & 0.02 & 0.02 & \\
\hline Fireplace & & 0.02 & 0.04 & & \\
\hline $\begin{array}{c}\text { Portable } \\
\text { Heater }\end{array}$ & 0.02 & & & \\
\hline Chimney & & & & & \\
\hline
\end{tabular}

ALL NON-RURAL 
Table 13. Heating fire fatality rates - HIGH/LOW states

(Form of material ignited: walls, ceiling, framing, insulation, flooring)

\begin{tabular}{|c|c|c|c|c|c|}
\hline EQP FHI & $\begin{array}{l}\text { GAS } \\
\text { FUEL }\end{array}$ & $\begin{array}{c}\text { LIQUID } \\
\text { FUEL }\end{array}$ & $\begin{array}{l}\text { SOLID } \\
\text { FUEL } \\
\end{array}$ & $\begin{array}{l}\text { ELEC- } \\
\text { TRIC } \\
\end{array}$ & OTHER \\
\hline $\begin{array}{l}\text { Central } \\
\text { Heating }\end{array}$ & 0.52 & & & & \\
\hline \multicolumn{6}{|l|}{$\begin{array}{l}\text { Water } \\
\text { Heater }\end{array}$} \\
\hline $\begin{array}{l}\text { Local } \\
\text { Heater }\end{array}$ & 0.13 & & 1.31 & 0.13 & \\
\hline Fireplace & & & 0.65 & & \\
\hline $\begin{array}{l}\text { Portable } \\
\text { Hea ter }\end{array}$ & & 0.26 & 0.13 & 0.13 & \\
\hline Chimney & & & 0.52 & & \\
\hline
\end{tabular}

HIGH RURAL

\begin{tabular}{|l|l|l|l|l|l|}
\hline ERP FHI & $\begin{array}{c}\text { GAS } \\
\text { FUEL }\end{array}$ & $\begin{array}{c}\text { LIQUID } \\
\text { FUEL }\end{array}$ & $\begin{array}{c}\text { SOLID } \\
\text { FUEL }\end{array}$ & $\begin{array}{c}\text { ELEC- } \\
\text { TRIC }\end{array}$ & OTHER \\
\hline $\begin{array}{l}\text { Central } \\
\text { Heating }\end{array}$ & & & & & \\
\hline $\begin{array}{l}\text { Water } \\
\text { Heater }\end{array}$ & 0.94 & & 1.89 & & \\
\hline $\begin{array}{l}\text { Local } \\
\text { Heater }\end{array}$ & & 0.71 & 1.18 & & \\
\hline $\begin{array}{l}\text { Fireplace } \\
\begin{array}{l}\text { Portable } \\
\text { Heater }\end{array}\end{array}$ & & & & & \\
\hline \begin{tabular}{l} 
Chimney \\
\hline
\end{tabular} & & & 0.71 & & \\
\hline
\end{tabular}

LOW RURAL

\begin{tabular}{|c|c|c|c|c|c|}
\hline EQP FHI & $\begin{array}{c}\text { GAS } \\
\text { FUEL }\end{array}$ & $\begin{array}{c}\text { LIQUID } \\
\text { FUEL }\end{array}$ & $\begin{array}{c}\text { SOLID } \\
\text { FUEL }\end{array}$ & $\begin{array}{c}\text { ELEC- } \\
\text { TRIC }\end{array}$ & OTHER \\
\hline $\begin{array}{c}\text { Central } \\
\text { Heating }\end{array}$ & & & 0.08 & \\
\hline $\begin{array}{c}\text { Water } \\
\text { Heater }\end{array}$ & & & 0.54 & 0.15 & \\
\hline $\begin{array}{c}\text { Local } \\
\text { Heater }\end{array}$ & 0.62 & & 0.38 & & \\
\hline $\begin{array}{c}\text { Fireplace } \\
\text { irepable }\end{array}$ & 0.08 & & 0.08 & & \\
\hline $\begin{array}{c}\text { Heater } \\
\text { Chimney }\end{array}$ & 0.08 & & 0.08 & \\
\hline
\end{tabular}

HIGH NON-RURAL

\begin{tabular}{|l|l|l|l|l|l|}
\hline EQP FHI & $\begin{array}{c}\text { GAS } \\
\text { FUEL }\end{array}$ & $\begin{array}{c}\text { LIQUID } \\
\text { FUEL }\end{array}$ & $\begin{array}{c}\text { SOLID } \\
\text { FUEL }\end{array}$ & $\begin{array}{c}\text { ELEC- } \\
\text { TRIC }\end{array}$ & OTHER \\
\hline $\begin{array}{c}\text { Central } \\
\text { Heating }\end{array}$ & & & 0.03 & \\
\hline $\begin{array}{c}\text { Water } \\
\text { Heater }\end{array}$ & & & & & \\
\hline $\begin{array}{c}\text { Local } \\
\text { Heater }\end{array}$ & 0.08 & & & & \\
\hline $\begin{array}{c}\text { Fireplace } \\
\text { Portable } \\
\text { Heater }\end{array}$ & & & & 0.06 & \\
\hline Chimney & & & & & \\
\hline
\end{tabular}

LOW NON-RURAL

Table 14. Heating fire fatality rates - HIGH/LOW states

(Form of material ignited: furniture, bedding, soft goods, trash)

\begin{tabular}{|c|c|c|c|c|c|}
\hline EQP FHI & $\begin{array}{l}\text { GAS } \\
\text { FUEL }\end{array}$ & $\begin{array}{c}\text { LIQUID } \\
\text { FUEL }\end{array}$ & $\begin{array}{l}\text { SOLID } \\
\text { FUEL } \\
\end{array}$ & $\begin{array}{l}\text { ELEC- } \\
\text { TRIC }\end{array}$ & OTHER \\
\hline \multicolumn{6}{|l|}{$\begin{array}{l}\text { Central } \\
\text { Heating }\end{array}$} \\
\hline \multicolumn{6}{|l|}{$\begin{array}{l}\text { Water } \\
\text { Heater }\end{array}$} \\
\hline $\begin{array}{l}\text { Local } \\
\text { Heater }\end{array}$ & 0.39 & & 0.26 & 0.52 & \\
\hline Fireplace & & & 0.65 & & \\
\hline $\begin{array}{c}\text { Portable } \\
\text { Heater }\end{array}$ & 0.13 & 0.13 & & 0.26 & \\
\hline Chimney & & & & & \\
\hline
\end{tabular}

HIGH RURAL

\begin{tabular}{|l|l|l|l|l|l|}
\hline EQP FHI & $\begin{array}{c}\text { GAS } \\
\text { FUEL }\end{array}$ & $\begin{array}{c}\text { LIQUID } \\
\text { FUEL }\end{array}$ & $\begin{array}{c}\text { SOLID } \\
\text { FUEL }\end{array}$ & $\begin{array}{c}\text { ELEC- } \\
\text { TRIC }\end{array}$ & OTHER \\
\hline $\begin{array}{c}\text { Central } \\
\text { Heating }\end{array}$ & & & & & \\
\hline $\begin{array}{l}\text { Water } \\
\text { Heater }\end{array}$ & & & & & \\
\hline $\begin{array}{l}\text { Local } \\
\text { Heater }\end{array}$ & & & 0.47 & & \\
\hline $\begin{array}{l}\text { Fireplace } \\
\text { Portable } \\
\text { Heater }\end{array}$ & & & & 0.94 & \\
\hline \begin{tabular}{l} 
Chimney \\
\hline
\end{tabular} & & & & & \\
\hline
\end{tabular}

LOW RURAL

\begin{tabular}{|c|c|c|c|c|c|}
\hline EQP FHI & $\begin{array}{c}\text { GAS } \\
\text { PUEL }\end{array}$ & $\begin{array}{c}\text { LIQUID } \\
\text { FUEL }\end{array}$ & $\begin{array}{c}\text { SOLID } \\
\text { FUEL }\end{array}$ & $\begin{array}{c}\text { ELEC- } \\
\text { TRIC }\end{array}$ & OTHER \\
\hline $\begin{array}{c}\text { Central } \\
\text { Heating }\end{array}$ & & & & & \\
\hline $\begin{array}{c}\text { Water } \\
\text { Heater }\end{array}$ & & & 0.23 & & \\
\hline $\begin{array}{c}\text { Local } \\
\text { Heater }\end{array}$ & 0.69 & & 0.15 & & \\
\hline $\begin{array}{c}\text { Fireplace } \\
\text { Portable } \\
\text { Heater }\end{array}$ & 0.08 & & & 0.62 & \\
\hline Chimney & & & & & \\
\hline
\end{tabular}

HIGH NON-RURAL

\begin{tabular}{|c|c|c|c|c|c|}
\hline EQP FHI & $\begin{array}{l}\text { GAS } \\
\text { FUEL }\end{array}$ & $\begin{array}{c}\text { LIQUID } \\
\text { FUEI }\end{array}$ & $\begin{array}{l}\text { SOLID } \\
\text { EUEL }\end{array}$ & $\begin{array}{l}\text { ELEC- } \\
\text { TBIC }\end{array}$ & OTHER \\
\hline \multicolumn{6}{|l|}{$\begin{array}{l}\text { Central } \\
\text { Heating }\end{array}$} \\
\hline $\begin{array}{l}\text { Water } \\
\text { Heater }\end{array}$ & 0.03 & & & & \\
\hline $\begin{array}{l}\text { Local } \\
\text { Heater }\end{array}$ & 0.11 & & & & \\
\hline \multicolumn{6}{|l|}{ Fireplace } \\
\hline $\begin{array}{c}\text { Portable } \\
\text { Heater }\end{array}$ & & 0.03 & & 0.28 & \\
\hline Chimney & & & & & \\
\hline
\end{tabular}

LOW NON-RURAL 
Table 15. Heating fire fatality rates - HIGH/LOW states

(Form of material ignited: garment on person)

\begin{tabular}{|l|l|l|l|l|l|}
\hline EQP FHI & GAS & $\begin{array}{c}\text { LIQUID } \\
\text { FUEL }\end{array}$ & $\begin{array}{l}\text { SOLID } \\
\text { FUEL }\end{array}$ & $\begin{array}{c}\text { ELEC- } \\
\text { TRIC }\end{array}$ & OTHER \\
\hline $\begin{array}{l}\text { Central } \\
\text { Heating }\end{array}$ & & & & & \\
\hline $\begin{array}{l}\text { Water } \\
\text { Heater }\end{array}$ & & & 0.26 & & 0.13 \\
\hline $\begin{array}{l}\text { Local } \\
\text { Heater }\end{array}$ & 1.05 & & 1.31 & & \\
\hline $\begin{array}{l}\text { Fireplace } \\
\text { Portable } \\
\text { Heater }\end{array}$ & & & & 0.26 & 0.13 \\
\hline Chimney & & & & & \\
\hline
\end{tabular}

HIGH RURAL

\begin{tabular}{|c|c|c|c|c|c|}
\hline EQP FHI & $\begin{array}{l}\text { GAS } \\
\text { FUEL } \\
\end{array}$ & $\begin{array}{c}\text { LIQUID } \\
\text { FUEI }\end{array}$ & $\begin{array}{l}\text { SOLID } \\
\text { EUEL }\end{array}$ & $\begin{array}{l}\text { ELEC- } \\
\text { TRIC }\end{array}$ & OTHER \\
\hline $\begin{array}{l}\text { Central } \\
\text { Heating }\end{array}$ & & - & & & \\
\hline \multicolumn{6}{|l|}{$\begin{array}{l}\text { Water } \\
\text { Heater }\end{array}$} \\
\hline $\begin{array}{l}\text { Local } \\
\text { Heater }\end{array}$ & & & 0.24 & . & . \\
\hline Fireplace & & & 0.47 & & \\
\hline \multicolumn{6}{|l|}{$\begin{array}{l}\text { Portable } \\
\text { Heater }\end{array}$} \\
\hline Chimney & & & & & \\
\hline
\end{tabular}

LOK RURAL

\begin{tabular}{|c|c|c|c|c|c|}
\hline EQP FHI & $\begin{array}{l}\text { GAS } \\
\text { FUEL }\end{array}$ & $\begin{array}{c}\text { LIQUID } \\
\text { FUEL }\end{array}$ & $\begin{array}{c}\text { SOLID } \\
\text { FUEL }\end{array}$ & $\begin{array}{c}\text { ELEC- } \\
\text { TRIC }\end{array}$ & OTHER \\
\hline $\begin{array}{c}\text { Central } \\
\text { Heating }\end{array}$ & & & & & \\
\hline $\begin{array}{c}\text { Water } \\
\text { Heater }\end{array}$ & & & 0.08 & 0.08 & \\
\hline $\begin{array}{c}\text { Local } \\
\text { Heater }\end{array}$ & 0.62 & & 0.08 & & \\
\hline $\begin{array}{c}\text { Fireplace } \\
\text { Pireatable } \\
\text { Peater }\end{array}$ & & & & & \\
\hline Chimney & & & & & \\
\hline
\end{tabular}

HIGH NON-RURAL

\begin{tabular}{|l|l|l|l|l|l|}
\hline EQP FHI & GAS & LIQUID & $\begin{array}{c}\text { SOLID } \\
\text { FUEI }\end{array}$ & $\begin{array}{c}\text { ELEC- } \\
\text { TRIC }\end{array}$ & OTHER \\
\hline $\begin{array}{c}\text { Central } \\
\text { Heating }\end{array}$ & & & & & \\
\hline $\begin{array}{c}\text { Water } \\
\text { Heater }\end{array}$ & & & & & \\
\hline $\begin{array}{c}\text { Local } \\
\text { Heater }\end{array}$ & 0.06 & & 0.03 & & \\
\hline $\begin{array}{c}\text { Fireplace } \\
\text { Portable } \\
\text { Heater }\end{array}$ & 0.03 & 0.09 & & 0.03 & \\
\hline Chimney & & & & & \\
\hline
\end{tabular}

LON NON-RURAL

Table 16. Heating fire fatality rates - HIGH/LOW states

(Form of material ignited: flammable liquid, fuel)

\begin{tabular}{|c|c|c|c|c|c|}
\hline EQP FHI & $\begin{array}{l}\text { GAS } \\
\text { FUEL } \\
\end{array}$ & $\begin{array}{l}\text { LIQUID } \\
\text { FUEL }\end{array}$ & $\begin{array}{l}\text { SOLID } \\
\text { FUEL }\end{array}$ & $\begin{array}{l}\text { ELEC- } \\
\text { TRIC }\end{array}$ & OTHER \\
\hline \multicolumn{6}{|l|}{$\begin{array}{l}\text { Central } \\
\text { Heating }\end{array}$} \\
\hline $\begin{array}{l}\text { Water } \\
\text { Heater }\end{array}$ & 0.26 & & & & \\
\hline $\begin{array}{l}\text { Local } \\
\text { Heater }\end{array}$ & 0.13 & & 0.39 & & 0.13 \\
\hline Fireplace & & & 0.13 & & 0.13 \\
\hline $\begin{array}{c}\text { Portable } \\
\text { Heater }\end{array}$ & 0.13 & 0.13 & & & \\
\hline Chimney & & & & & \\
\hline
\end{tabular}

HIGH RURAI

\begin{tabular}{|c|c|c|c|c|c|}
\hline EQP FHI & GAS & $\begin{array}{c}\text { LIQUID } \\
\text { FUEL }\end{array}$ & $\begin{array}{c}\text { SOLID } \\
\text { FUEI }\end{array}$ & $\begin{array}{c}\text { ELEC- } \\
\text { TRIC }\end{array}$ & OrHER \\
\hline $\begin{array}{c}\text { Central } \\
\text { Heating }\end{array}$ & & & & & \\
\hline $\begin{array}{c}\text { Water } \\
\text { Heater }\end{array}$ & 0.31 & 0.15 & 0.08 & & 0.08 \\
\hline $\begin{array}{c}\text { Local } \\
\text { Heater }\end{array}$ & 0.23 & 0.08 & & & \\
\hline \begin{tabular}{c} 
Fireplace \\
\hline $\begin{array}{c}\text { Portable } \\
\text { Heater }\end{array}$
\end{tabular} & 0.08 & 0.08 & & \\
\hline Chimney & & HIGH NON-RURAL & & \\
\hline
\end{tabular}

HIGH NON-RURAL

\begin{tabular}{|l|l|l|l|l|l|}
\hline EQP FHI & $\begin{array}{c}\text { GAS } \\
\text { FUEL }\end{array}$ & $\begin{array}{c}\text { LIQUID } \\
\text { FUEL }\end{array}$ & $\begin{array}{c}\text { SOLID } \\
\text { FUEL }\end{array}$ & $\begin{array}{c}\text { ELEC- } \\
\text { TRIC }\end{array}$ & OTHER \\
\hline $\begin{array}{l}\text { Central } \\
\text { Heating }\end{array}$ & & & & & \\
\hline $\begin{array}{l}\text { Water } \\
\text { Heater }\end{array}$ & 0.94 & & & & \\
\hline $\begin{array}{l}\text { Local } \\
\text { Heater }\end{array}$ & & & 0.47 & & 0.24 \\
\hline $\begin{array}{l}\text { Fireplace } \\
\begin{array}{c}\text { Portable } \\
\text { Heater }\end{array}\end{array}$ & & & & & \\
\hline \begin{tabular}{l} 
Chimney \\
\hline
\end{tabular} & & & & & \\
\hline
\end{tabular}

LOW RURAL

\begin{tabular}{|l|c|c|c|c|c|}
\hline EQP FHI & $\begin{array}{c}\text { GAS } \\
\text { FUEL }\end{array}$ & $\begin{array}{c}\text { LIQUID } \\
\text { FUEL }\end{array}$ & $\begin{array}{c}\text { SOLID } \\
\text { FUEL }\end{array}$ & $\begin{array}{c}\text { ELEC- } \\
\text { TRIC }\end{array}$ & OTHER \\
\hline $\begin{array}{c}\text { Central } \\
\text { Heating }\end{array}$ & & & & & \\
\hline $\begin{array}{c}\text { Water } \\
\text { Heater }\end{array}$ & 0.33 & 0.11 & 0.03 & 0.03 & 0.03 \\
\hline $\begin{array}{c}\text { Local } \\
\text { Heater }\end{array}$ & 0.36 & 0.03 & & \\
\hline $\begin{array}{c}\text { Fireplace } \\
\text { Portable } \\
\text { Heater }\end{array}$ & & 0.0 & & \\
\hline Chimney & & & & & \\
\hline
\end{tabular}

LOW NON-RURAL 
RURAI Fatality Rate NON-RURAL Fatality Rate

\subsection{3 fatalities/million}

0.26 fatalities/million

Inadequate separation between solid fueled (usually wood fueled) heating stoves or fireplaces and combustible structural components results in this scenario. Typically a wood stove is located too close to a wood paneled wall, which then ignites from the stove's heat. Heat from vents or chimneys installed with inadequate clearance often ignites wall coverings or the framing inside the wall. Also included in the scenario are cases where flooring or floor covering was not adequately protected against the heat or flames from a stove or fireplace and subsequently ignited.

Table 13 shows that RURAI areas in HIGH and LOW states have similar fatality rates for this scenario, significantly higher than the NON-RURAL rates, indicating that it is primarily a RURAI fire problem. In the NON-RURAL areas, the only fatalities for this scenario occurred in the HIGH states, although the fatality rate is lower than the RURAI rate in either HIGH or LOW states.

The following causal factors define this scenario:

Equipment: Local heater, fireplace, chimney Form of Heat: Solid fueled equipment Form of Material Ignited: Wall, ceiling, framing, insulation, floor The fatality rates for this scenario are taken from table 9.

2. COMBUSTIBLES PLACED TOO CLOSE TO SOLID FUEIED HEATING EQUIPMENT

RURAL Fatality Rate NON-RURAI Fatality Rate
0.76 fatalities/million

0.10 fatalities/million

Wood stoves and fireplaces often provided the only heat in RURAL dwellings where this scenario occurred. Typically occupants placed a bed or chair too close to the heating equipment in order to keep warm, and the bed or chair subsequently was ignited by heat or flame escaping from the stove while the occupants were sleeping.

Predominating this scenario is the HIGH RURAI fatality rate (table 14), with LOW RURAI and HIGH NON-RURAI areas having similar rates, indicating both a RURAL and a HIGH state fire problem.

The following causal factors define this scenario:

Equipment: Local heater, fireplace Form of Heat: Solid fueled equipment Form of Material Ignited: Furniture, bedding, soft goods, trash The fatality rates for this scenario are taken from table 10.

\section{FLAMMABLE LIQUID USED TO KINDLE FIRE}
RURAL Fatality Rate
0.50 fatalities/million
NON-RURAL Fatality Rate
0.02 fatalities/million

Persons involved in this scenario used flammable liquids to start fires in fireplaces and wood stoves. A flash fire then resulted, often involving excessive or spilled flammable liquid, which burned the involved person. Persons involved in this scenario were often coded on the worksheet as being impaired by alcohol. 
RURAL areas in HIGH and LOW states have similar fatality rates for this scenario (table 16) and are significantly higher than either NON-RURAL rate, indicating that this scenario is primarily RURAL in scope.

The following causal factors define this scenario:

Equipment: Local heater, fireplace

Form of Heat: Solid fueled equipment

Form of Material Ignited: Flammable liquid, fuel

The fatality rates for this scenario are taken from table 12.

RURAI Fatality Rate NON-RURAL Fatality Rate
2.26 fatalities/million

0.38 fatalities $/ \mathrm{million}$

Elderly people were often the victims of this scenario. Typically, they were sitting close to wood stoves and local space heaters to keep warm when their clothing ignited. Fires of this type were often not reported to the fire department, as the victim was transported to the hospital by relatives or neighbors. This type of fire fequently involved only the clothing on the victim. Wood stoves were the most common ignition source.

The highest rates in this scenario are those in the HIGH RURAI areas, as indicated in table 15, with the heating equipment involved primarily divided between gas fueled heaters and solid fueled heating equipment. IOW RURAI and HIGH NON-RURAI areas had similar fatality rates for this scenario, about 25 percent of the HIGH RURAL rate, followed by LOW NON-RURAL areas with a rate less than $D$ percent of the HIGH RURAI rate. Gas fueled equipment appears more significant in HIGH states than in LOW states, while solid fueled equipment is more significant in RURAL than NON-RURAL areas.

The following causal factors define this scenario:

Equipment: All heating equipment

Form of Heat: All

Form of Material Ignited: Garment on person

The fatality rates for this scenario are taken from table 11.

\section{PORTABLE SPACE HEATERS}

RURAI Fatality Rate NON-RURAL Fatality Rate
1.66 fatalities/million

0.48 fatalities/million

This scenario involves the misuse of portable space heaters, primarily liquid fueled, solid fueled, and electric. Ignition occurs when the heater is placed too close to combustibles, primarily wall paneling, bedding or upholstered furniture. The portable heaters are often used in this scenario as a substitute for central or stationary local heating. Most of the differences between the RURAI and the NON-RURAL fatality rates for this scenario are accounted for to the ignition of wood paneling by solid and liquid fueled portable heaters.

Portable heater fatality rates are highest in RURAL areas of IOW states by a significant amount (tables 13 and 14). Liquid fueled equipment and solid fueled equipment igniting combustible walls in RURAL areas, and electric equipment igniting fabric in all areas contribute most significantly to the overall rate for this scenario.

The following causal factors define this scenario:

Equipment: Portable heater

Form of Heat: All

Form of Material Ignited: Walls, floors, furniture, bedding, soft goods, The fatality rates for this scenario are taken from tables 9 and 10. 
$\begin{array}{ll}\text { RURAL Fatality Rate } & 0.68 \text { fatalities/million }{ }^{5} \\ \text { NON-RURAL Fatality Rate } & 0.00\end{array}$

This scenario represents the ignition of the wood wall paneling in gas fired furnace and water heater enclosures in mobile homes. older mobile homes, which are particularly common in RURAL areas; usually do not provide any fire rated enclosure around furnaces and water heaters, which are separated from the living unit by combustible paneling only.

As indicated in table 13, the RURAL fatality rate for this scenario consisted of central heating systems in HIGH states and water heaters in LOW states. It is not known if any significance can be attached to this distribution.

The following causal factors define this scenario:

Equipment: Central heating, water heater Form of Heat: Gas fueled equipment Form of Material Ignited: Walls, ceilings, framing; insulations, flooring The fatality rates for this scenario are taken from table 9.

Overall, solid fueled heating equipment in RURAL areas of both HIGH and LOW states is by far the leading contributor to heating fire fatalities. Table 17 ranks heating equipment and area category. Structural materials are the most common material ignited.

Table 17. Heating fire fatality rates ranked by equipment and area category

\begin{tabular}{rlll}
\hline Rank & Area Category & Equipment & Fatality Rate \\
\hline 1 & HIGH RURAL & Solid Fuel & 8.24 \\
2 & LOW RURAL & Solid Fuel & 5.42 \\
3 & HIGH RURAL & Gas Fuel & 4.05 \\
4 & HIGH NON-RURAL & Gas Fuel & 3.31 \\
5 & LOW RURAL & Gas Fuel & 1.89 \\
6 & HIGH NON-RURAL & Solid Fuel & 1.85 \\
7 & HIGH RURAL & Electric & 1.31 \\
8 & HIGH NON-RURAL & Electric & 1.00 \\
9 & LOW RURAL & Electric & 0.94 \\
10 & LOW RURAL & Liquid Fuel & 0.94 \\
11 & LOW NON-RURAL & Gas Fuel & 0.85 \\
12 & HIGH RURAL & Liquid Fuel & 0.78 \\
13 & LOW NON-RURAL & Liquid Fuel & 0.50 \\
14 & LOW NON-RURAL & Electric & 0.41 \\
15 & HIGH NON-RURAL & Liquid Fuel & 0.23 \\
16 & LOW NON-RURAL & Solid Fuel & 0.11
\end{tabular}

The mean fatality rate for this data set is 1.99 .

${ }^{\mathrm{A} I 1}$ in mobile homes. 


\subsection{Cooking Fire Scenarios}

The fatality rate in RURAL cooking fires (4.2) is triple the rate for NON-RURAL cooking fires $(1.4)$. Table 18 shows the RURAL and NON-RURAL cooking fatality rates for ALL states by Type of Material Ignited (TMI) and Ignition Factor (IF). Table 19 provides the same breakdown by RURAL and URBAN areas for both HIGH and LOW states.

Table 18. Cooking fire fatality rates - ALL states

(Form of heat: all (75\% gas fueled equipment))

\begin{tabular}{|c|c|c|c|c|c|}
\hline IF TMI & GAS & $\begin{array}{l}\text { FLAY1. } \\
\text { LIQUID }\end{array}$ & $\begin{array}{l}\text { COOK } \\
\text { MTL. }\end{array}$ & WALLS & FABRIC \\
\hline Unattended & & & 1.10 & & \\
\hline $\begin{array}{l}\text { Misuse of } \\
\text { Flammables }\end{array}$ & & 0.25 & & & \\
\hline $\begin{array}{l}\text { Misuse of } \\
\text { Fuel }\end{array}$ & 0.34 & 0.08 & & & \\
\hline $\begin{array}{l}\text { Combustible } \\
\text { Too Close }\end{array}$ & & & & & 0.59 \\
\hline $\begin{array}{l}\text { Installation, } \\
\text { Maintenance }\end{array}$ & 0.42 & & & 0.08 & \\
\hline $\begin{array}{l}\text { Operational } \\
\text { Problem }\end{array}$ & 0.08 & & & & \\
\hline
\end{tabular}

\begin{tabular}{|l|l|l|l|l|l|}
\hline IF TMI & GAS & $\begin{array}{c}\text { FLAM. } \\
\text { LIQUID }\end{array}$ & $\begin{array}{c}\text { COOK } \\
\text { MTL. }\end{array}$ & WALLS & FABRIC \\
\hline Unattended & & 0.10 & & 0.06 & \\
\hline $\begin{array}{l}\text { Misuse of } \\
\text { Flammables }\end{array}$ & 0.06 & 0.02 & & & \\
\hline $\begin{array}{l}\text { Misuse of } \\
\text { Fuel }\end{array}$ & & & & & 0.49 \\
\hline $\begin{array}{c}\text { Combustible } \\
\text { Too close }\end{array}$ & 0.02 & & & & \\
\hline $\begin{array}{c}\text { Installation, } \\
\text { Maintenance }\end{array}$ & & & & & \\
\hline $\begin{array}{c}\text { Operational } \\
\text { Problem }\end{array}$ & 0.22 & & \\
\hline
\end{tabular}

ALL RURAL

ALL NON-RURAL

Table 19. Cooking fire fatality rates - HIGH/LOW states (Form of heat: 11 (75\% gas fueled equipment))

\begin{tabular}{|c|c|c|c|c|c|}
\hline IF TMI & GAS & $\begin{array}{l}\text { FLAIM. } \\
\text { LIQUID }\end{array}$ & $\begin{array}{l}\text { COOK } \\
\text { MTL. }\end{array}$ & WALLS & FABRIC \\
\hline Unattended & & & 1.57 & 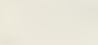 & \\
\hline $\begin{array}{l}\text { Misuse of } \\
\text { Flammables }\end{array}$ & & 0.26 & & & \\
\hline $\begin{array}{l}\text { Misuse of } \\
\text { Fuel }\end{array}$ & & 0.13 & & & \\
\hline $\begin{array}{l}\text { Combustible } \\
\text { Too Close }\end{array}$ & & & & & 0.26 \\
\hline $\begin{array}{l}\text { Installation, } \\
\text { Maintenance }\end{array}$ & 0.52 & & & & \\
\hline $\begin{array}{l}\text { Pperational } \\
\text { Problem }\end{array}$ & 0.13 & & & & \\
\hline
\end{tabular}

EIGH RURAL

\begin{tabular}{|c|l|l|l|l|l|}
\hline IF TMI & GAS & $\begin{array}{l}\text { FLAM. } \\
\text { LIQUID }\end{array}$ & $\begin{array}{c}\text { COOK } \\
\text { MTL. }\end{array}$ & WALLS & FABRIC \\
\hline Unattended & & & 1.65 & 0.47 & \\
\hline $\begin{array}{c}\text { Misuse of } \\
\text { Flammables }\end{array}$ & 0.71 & & & \\
\hline $\begin{array}{c}\text { Misuse of } \\
\text { Fuel }\end{array}$ & 0.47 & & & & \\
\hline $\begin{array}{c}\text { Combustible } \\
\text { Too close }\end{array}$ & & & & & 1.41 \\
\hline $\begin{array}{c}\text { Installation, } \\
\text { Maintenance }\end{array}$ & & & & & \\
\hline $\begin{array}{c}\text { Operational } \\
\text { Problem }\end{array}$ & & & & & \\
\hline
\end{tabular}

Problem

HIGH NON-RURAL

\begin{tabular}{|l|l|l|l|l|l|}
\hline IF TMI & GAS & $\begin{array}{l}\text { FLAM. } \\
\text { LISUID }\end{array}$ & $\begin{array}{c}\text { COOK } \\
\text { MTL }\end{array}$ & WALLS & FABRIC \\
\hline $\begin{array}{l}\text { Unattended } \\
\begin{array}{l}\text { Misuse of } \\
\text { Flammables }\end{array}\end{array}$ & & 0.24 & 0.24 & \\
\hline $\begin{array}{l}\text { Misue of } \\
\text { Fuel }\end{array}$ & 0.94 & & & & \\
\hline $\begin{array}{c}\text { Combustibles } \\
\text { Too Close }\end{array}$ & & & & & 1.18 \\
\hline $\begin{array}{c}\text { Installation, } \\
\text { Maintenance }\end{array}$ & 0.24 & & & 0.24 & \\
\hline $\begin{array}{c}\text { Operational } \\
\text { Problem }\end{array}$ & & & & & \\
\hline
\end{tabular}

\begin{tabular}{|c|c|c|c|c|c|}
\hline IF TMI & GAS & $\begin{array}{l}\text { FLAM. } \\
\text { LIQUID }\end{array}$ & $\begin{array}{l}\text { COOKK } \\
\text { MTL. }\end{array}$ & WALLS & FABRIC \\
\hline Unattended & & & 0.11 & 0.03 & \\
\hline $\begin{array}{l}\text { Misuse of } \\
\text { Flammables }\end{array}$ & & 0.06 & & & \\
\hline $\begin{array}{l}\text { Misuse of } \\
\text { Fuel }\end{array}$ & 0.03 & 0.03 & & & \\
\hline $\begin{array}{l}\text { Combustibles } \\
\text { Too Close }\end{array}$ & & & & & 0.39 \\
\hline $\begin{array}{l}\text { Installation, } \\
\text { Maintenance }\end{array}$ & & & & & \\
\hline $\begin{array}{l}\text { Operational } \\
\text { Problem }\end{array}$ & 0.03 & & & & \\
\hline
\end{tabular}


The differences in cooking fire fatality rates between RURAL and NON-RURAL areas appears primarily related to three basic scenarios. About 75 percent of the cooking fire fatalities involved gas fueled equipment in both RURAI and NON-RURAI areas.

\section{UNATTENDED COOKING}

\section{RURAL Fatality Rate} NON-RURAL Fatality Rate

\subsection{8 fatalities/million \\ 0.28 fatalities/million}

The largest discrepancy between the RURAL and NON-RURAL cooking fatality rates was related to unattended cooking. Intoxication contributed to about 35 percent of the unattended cooking fatalities in both RURAI and NON-RURAI areas. In the majority of cases cooking materials (food or grease) ignited while the occupant(s) were asleep or incapacitated in another room. As seen in table 19, the bulk of this RURAL fire problem is in the HIGH states. In fact, the NON-RURAL areas of the HIGH states also have a high unattended cooking fatality rate, while the rate for both RURAL and NON-RURAI areas in the LOW states is considerably lower.

The following causal factors define this scenario:

Equipment: Cooking equipment, all fuels Type Material Ignited: Cooking material, walls (hardboard, plywood) Ignition Factor: Unattended (includes falling asleep, unconscious)

2. INSTALLATION/MAINTENANCE DEFICIENCIES
RURAL Fatality Rate NON-RURAL Fatality Rate
0.50 fatalities/million
0.00 fatalities/million

Gas fuel leaks dominate this RURAL fatality scenario, most often involving Liquified Petroleum Gas (LPG). While specific deficiencies are not usually identified, this scenario appears to relate significantly to the widespread use of LPG in RURAL areas in both HIGH and LOW states.

The following causal factors define this scenario:

Equipment: Cooking equipment, all fuels

Type Material Ignited: Gas, walls

Ignition Factor: Installation/maintenance (leak, part failure, break, installation deficiency)

3. MISUSE OF FLAMMABLE LIQUIDS, GASES
RURAL Fatality Rate
NON-RURAL Fatality Rate
0.67 fatalities/million
0.18 fatalities/million

This scenario has two main components as indicated in table 18. The first, involving misuse of non-fuel flammable liquids, primarily consists of cleaning parts, stripping floors, etc. using flammable liquids which are ignited by stove pilot lights, or using flammable liquids as kindling for wood stoves.

The second component involves misuse of fuel, primarily improper fueling or lighting of gas stoves or accidental release of fuel.

As can be seen in table 19, the misuse of flammable liquids also appears to be a significant problem in the NON-RURAL areas of HIGH states, while the misuse of fuel problem occurs primarily in the RURAL areas of LOW states.

The following causal factors define this scenario:

Equipment: Cooking equipment, all fuels

Type Material Ignited: Gas, flammable liquid

Ignition Factor: Misuse of fuel (accidental release, spill, improper fueling) 
Table 18 also indicates a substantial fabric too close to heat fatality rate in both RURAL and NON-RURAL areas. These are primarily clothing ignitions, often involving the elderly. Table 19 indicates that this problem is most severe in the RURAL areas of LOW states, and the NON-RURAL areas of HIGH states.

\subsection{Smoking Fire Scenarios}

The fatality rate in RURAL smoking fires (7.3) is 25 percent higher than the NON-RURAL smoking fire fatality rate (5.9). Table 20 shows the RURAL and NON-RURAL smoking fatality rates (CBI) and Form of Material Ignited (FMI). The difference in fatality rates between RURAL and NON-RURAL areas do not appear from table 20 to be explained by any single factor. These differences can be described by two scenarios; with the number of intoxicated fatalities slightly higher in RURAL areas for both scenarios.

Table 20. Smoking fire fatality rates - ALI states (Cause: Smoking)

\begin{tabular}{|l|c|c|c|c|}
\hline CBI FMI & BEDDING & $\begin{array}{c}\text { UPHOL. } \\
\text { FURN. }\end{array}$ & OTHER & TOTAL \\
\hline Asleep & 1.77 & 1.60 & 0.08 & 3.45 \\
\hline Intoxicated & 1.09 & 1.09 & 0.17 & 2.35 \\
\hline Other & 0.34 & 0.34 & 0.17 & 0.85 \\
\hline Total & 3.20 & 3.03 & 0.42 & 6.65 \\
\hline
\end{tabular}

\begin{tabular}{|l|c|c|c|c|}
\hline \multicolumn{1}{|c|}{ CBI FMI } & BEDDING & $\begin{array}{l}\text { UPHOL. } \\
\text { FURN. }\end{array}$ & OTHER & TOTAL \\
\hline Asleep & 1.03 & 1.26 & 0.08 & 2.37 \\
\hline Intoxicated & 0.81 & 0.83 & 0.16 & 1.80 \\
\hline Other & 0.34 & 0.41 & 0.10 & 0.85 \\
\hline Total & 2.18 & 2.50 & 0.34 & 5.02 \\
\hline
\end{tabular}

ALL RURAL

ALL NON-RURAL

1. SMOKING IN BED

RURAL Fatality Rate NON-RURAL Fatality Rate
3.20 fatalities/million

2.18 fatalities/million

Smoking in bed dominates the RURAL/NON-RURAL fatality rate discrepancy in these fires. Intoxication is often a factor. Although the "intoxicated" fatality rate is slightly higher in RURAL areas, its contribution to the overall smoking in bed fatality rate is proportionately slightly higher in NONRURAL areas, possibly indicating that simple carelessness may be more of a factor in RURAL areas. Falling asleep with smoking materials dominates the smoking in bed scenario.

The following causal factors define this scenario:

Equipment: None

Form of Heat: Smoking materials

Form of Material Ignited: Mattress, bedding

2. SMOKING/UPHOLSTERED FURNITURE FIRES

RURAL Fatality Rate

NON-RURAL Fatality Rate
3.03 fatalities/million

2.50 fatalities/million

Smoking materials igniting upholstered furniture are also a more significant contributor to the fatality rate in RURAL areas than in NON-RURAL areas. The "intoxicated" fatality rate is again slightly higher in RURAL areas, but contributes about the same to the overall smoking/upholstered furniture fatality rate in both areas. The incidence of falling asleep with smoking materials (as opposed to discarding smoking materials) appears slightly higher in RURAL areas. 
The following causal factors define this scenario:

Equipment: None

Form of Heat: Smoking materials

Form of Material Ignited: Upholstered furniture

When smoking fires are examined by HIGH and LOW states, as well as RURAI and NON-RURAL areas, some interesting patterns emerge, as shown in table 21 . Smoking fire fatality rates are highest in LOW RURAL areas, followed by HIGH NON-RURAL, HIGH RURAL, and IOW NON-RURAL. Smoking while intoxicated is more prevalent in the HIGH than the IOW states, but does not show a significant RURAL/NON-RURAL pattern. Finally, the RURAL/NON-RURAL fatality rate discrepancy for smoking fires is primarily accounted for by the LOW RURAL fatality rate, which is dominated by upholstered furniture fires. This implies that while smoking in bed dominates RURAL smoking fire fatality rates in terms of number of fatalities, upholstered furniture fires in LOW states produce the most severe problem in terms of fatality rates.

Table 21. Smoking fire fatality rates - HIGH/LOW states

(Cause: Smoking)

\begin{tabular}{|l|c|c|c|l|}
\hline CBI FMI & BEDDING & $\begin{array}{c}\text { UPHOL } \\
\text { FURN }\end{array}$ & OTHER & TOTAL \\
\hline Asleep & 1.70 & 1.05 & & 2.75 \\
\hline Intoxicated & 1.57 & 1.05 & 0.13 & 2.75 \\
\hline Other & 0.13 & 0.26 & & 0.39 \\
\hline Total & 3.40 & 2.36 & 0.13 & 5.89 \\
\hline
\end{tabular}

\begin{tabular}{|l|c|c|c|c|}
\hline \multicolumn{1}{|c|}{ CBI FMI } & BEDDING & $\begin{array}{c}\text { UPHOL. } \\
\text { FURN. }\end{array}$ & OTHER & TOTAL \\
\hline Asleep & 1.61 & 1.31 & 0.08 & 3.00 \\
\hline Intoxicated & 1.08 & 1.54 & 0.15 & 2.77 \\
\hline Other & 0.69 & 0.54 & 0.23 & 1.46 \\
\hline Total & 3.38 & 3.39 & 0.46 & 7.23 \\
\hline
\end{tabular}

$\underline{\text { HIGH RURAL }}$

HIGH NON-RURAL

\begin{tabular}{|l|c|c|c|l|}
\hline CBI FMI & BEDDING & $\begin{array}{r}\text { UPHOL. } \\
\text { FURN. }\end{array}$ & OTHER & TOTAL \\
\hline Asleep & 1.89 & 2.59 & 0.24 & 4.72 \\
\hline Intoxicated & 0.24 & 1.18 & 0.24 & 1.66 \\
\hline Other & 0.71 & 0.47 & 0.47 & 1.65 \\
\hline Total & 2.84 & 4.24 & 0.96 & 8.04 \\
\hline
\end{tabular}

LOW RURAL

\begin{tabular}{|l|c|c|c|c|}
\hline \multicolumn{1}{|c|}{ CBI FMI } & BEDDING & $\begin{array}{c}\text { UPHOL. } \\
\text { FURN. }\end{array}$ & OTHER & TOTAL \\
\hline Asleep & 0.83 & 1.24 & 0.08 & 2.15 \\
\hline Intoxicated & 0.72 & 0.58 & 0.17 & 1.47 \\
\hline Other & 0.22 & 0.36 & 0.06 & 0.64 \\
\hline Total & 1.77 & 2.18 & 0.31 & 4.26 \\
\hline
\end{tabular}

LOW NON-RURAL

\subsection{Electrical Distribution Fire Scenarios}

The fatality rate in RURAL electrical distribution equipment fires (2.5) is over twice as high as the NON-RURAL rate (1.1). Tables 22-24 give the fatality rates for RURAL and NON-RURAL electrical distribution fires for ALL states by Equipment Involved in Ignition (EQP) and Form of Material Ignited (FMI) for one and two family dwellings, mobile homes and other residences (apartments, hotels, etc.). Most of the difference between RURAL and NON-RURAL fatality rates for electrical distribution fires is accounted for by a single scenario. Tables 25-27 gives the fatality rate distributions by HIGH and LOW states, as well as RURAL and NON-RURAL areas. 
Table 22. Electrical distribution fire fatality rates - ALL states (Fixed property use: one and two family)

\begin{tabular}{|l|c|c|c|c|}
\hline EQP FMI & $\begin{array}{c}\text { Wall } \\
\text { Celling }\end{array}$ & $\begin{array}{c}\text { Framing, } \\
\text { Insul. }\end{array}$ & $\begin{array}{c}\text { Bedding } \\
\text { Furn. }\end{array}$ & Other \\
\hline $\begin{array}{l}\text { Fixed Wiring } \\
\text { Switch, Recep. }\end{array}$ & 0.42 & 0.50 & & 0.08 \\
\hline $\begin{array}{l}\text { Cord, Plug } \\
\text { Bulb }\end{array}$ & 0.08 & & 0.25 & 0.08 \\
\hline Other & & & & \\
\hline
\end{tabular}

$\underline{\text { ALL RURAL }}$

\begin{tabular}{|l|c|c|c|c|}
\hline EQP FMI & $\begin{array}{c}\text { Wa11, } \\
\text { Celling }\end{array}$ & $\begin{array}{c}\text { Fram1ng, } \\
\text { Insul. }\end{array}$ & $\begin{array}{c}\text { Bedd1ng } \\
\text { Furn. }\end{array}$ & Other \\
\hline $\begin{array}{l}\text { Fixed Wirlng } \\
\text { Switch, Recep. }\end{array}$ & 0.16 & 0.10 & & 0.02 \\
\hline $\begin{array}{l}\text { Cord, Plug } \\
\text { Bulb }\end{array}$ & 0.12 & & 0.04 & 0.16 \\
\hline Other & & & & \\
\hline
\end{tabular}

ALL NON-RURAL

Table 23. Electrical distribution fire fatality rates - ALL states (Fixed property use: mobile homes)

\begin{tabular}{|l|c|c|c|c|}
\hline EQP FMI & $\begin{array}{c}\text { Wal1, } \\
\text { Ceiling }\end{array}$ & $\begin{array}{c}\text { Framing, } \\
\text { Insul. }\end{array}$ & $\begin{array}{c}\text { Bedding } \\
\text { Furn. }\end{array}$ & Other \\
\hline $\begin{array}{l}\text { Fixed Wiring } \\
\text { Switch, Recep }\end{array}$ & 0.34 & & & \\
\hline $\begin{array}{l}\text { Cord, Plug } \\
\text { Bulb }\end{array}$ & & & & \\
\hline Other & & & & \\
\hline
\end{tabular}

ALL RURAL

\begin{tabular}{|l|l|l|l|l|}
\hline EQP FMI & $\begin{array}{c}\text { Wall, } \\
\text { Ceiling }\end{array}$ & $\begin{array}{c}\text { Framing, } \\
\text { Insul. }\end{array}$ & $\begin{array}{c}\text { Bedding } \\
\text { Furn. }\end{array}$ & Other \\
\hline $\begin{array}{l}\text { Fixed Wiring } \\
\text { Switch, Recep. }\end{array}$ & & & & \\
\hline $\begin{array}{l}\text { Cord, Plug } \\
\text { Bulb }\end{array}$ & & & & 0.04 \\
\hline Other & & & & \\
\hline
\end{tabular}

ALL NON-RURAL

Table 24. Electrical distribution fire fatality rates - ALL states (Fixed property use: other residential)

\begin{tabular}{|l|r|r|r|r|}
\hline \multicolumn{1}{|c|}{ EQP FMI } & $\begin{array}{c}\text { Wall, } \\
\text { Celling }\end{array}$ & $\begin{array}{c}\text { Framing, } \\
\text { Insul. }\end{array}$ & $\begin{array}{c}\text { Bedding } \\
\text { Furn. }\end{array}$ & Other \\
\hline $\begin{array}{l}\text { Fixed Wiring } \\
\text { Switch, Recep }\end{array}$ & 0.08 & & & \\
\hline $\begin{array}{l}\text { Cord, Plug } \\
\text { Bulb }\end{array}$ & & & & \\
\hline Other & & & 0.08 & \\
\hline
\end{tabular}

ALL RURAL

\begin{tabular}{|l|c|c|r|r|}
\hline EQP FMI & $\begin{array}{c}\text { Wall, } \\
\text { Ceiling }\end{array}$ & $\begin{array}{c}\text { Framing, } \\
\text { Insul. }\end{array}$ & $\begin{array}{r}\text { Bedding } \\
\text { Furn. }\end{array}$ & Other \\
\hline $\begin{array}{l}\text { Fixed Wiring } \\
\text { Switch, Recep. }\end{array}$ & 0.02 & & 0.02 & \\
\hline $\begin{array}{l}\text { Cord, P1ug } \\
\text { Bulb }\end{array}$ & 0.06 & 0.06 & 0.02 & 0.10 \\
\hline Other & 0.06 & \\
\hline
\end{tabular}

ALL NON-RURAL 
Table 25. Electrical distribution fire fatality rates - HIGH/LOW states

(Fixed property use: one and two family)

\begin{tabular}{|l|c|c|c|c|}
\hline \multicolumn{1}{|c|}{ EQP } & $\begin{array}{c}\text { Wall, } \\
\text { Ceiling }\end{array}$ & $\begin{array}{c}\text { Framing, } \\
\text { Insul. }\end{array}$ & $\begin{array}{c}\text { Bedding } \\
\text { Furn. }\end{array}$ & Other \\
\hline $\begin{array}{l}\text { Fixed Wiring } \\
\text { Switch, Recep. }\end{array}$ & 0.65 & 0.78 & & 0.13 \\
\hline $\begin{array}{l}\text { Cord, Plug } \\
\text { Bulb }\end{array}$ & & & 0.13 &. \\
\hline Other & & & & \\
\hline
\end{tabular}

HIGH RURAL

\begin{tabular}{|l|c|c|c|c|}
\hline EQP FMI & $\begin{array}{c}\text { Wall, } \\
\text { Ceiling }\end{array}$ & $\begin{array}{c}\text { Framing, } \\
\text { Insul. }\end{array}$ & $\begin{array}{c}\text { Bedding } \\
\text { Furn. }\end{array}$ & Other \\
\hline $\begin{array}{l}\text { Fixed Wiring } \\
\text { Switch, Recep. }\end{array}$ & & & & \\
\hline $\begin{array}{l}\text { Cord, Plug } \\
\text { Bulb }\end{array}$ & 0.24 & & 0.47 & 0.24 \\
\hline Other & & & & \\
\hline
\end{tabular}

LOW RURAL

\begin{tabular}{|l|c|c|c|c|}
\hline EQP FMI & $\begin{array}{c}\text { Wall, } \\
\text { Ceiling }\end{array}$ & $\begin{array}{c}\text { Framing, } \\
\text { Insul. }\end{array}$ & $\begin{array}{c}\text { Bedding } \\
\text { Furn. }\end{array}$ & Other \\
\hline $\begin{array}{l}\text { Fixed Wiring } \\
\text { Switch, Recep. }\end{array}$ & 0.54 & 0.23 & & 0.08 \\
\hline $\begin{array}{l}\text { Cord, Plug } \\
\text { Bulb }\end{array}$ & 0.15 & & 0.15 & 0.15 \\
\hline Other & & & & \\
\hline
\end{tabular}

HIGH NON-RURAL

\begin{tabular}{|l|c|c|c|c|}
\hline EQP FMI & $\begin{array}{r}\text { Wall, } \\
\text { Ceiling }\end{array}$ & $\begin{array}{r}\text { Framing, } \\
\text { Insul. }\end{array}$ & $\begin{array}{c}\text { Bedding } \\
\text { Furn. }\end{array}$ & Other \\
\hline $\begin{array}{l}\text { Fixed Wiring } \\
\text { Switch, Recep. }\end{array}$ & 0.03 & 0.06 & & \\
\hline $\begin{array}{l}\text { Cord, Plug } \\
\text { Bulb }\end{array}$ & 0.11 & & & 0.17 \\
\hline Other & & & & \\
\hline
\end{tabular}

LOW NON-RURAL

Table 26. Electrical distribution fire fatality rates - HIGH/LOW states (Fixed property use: mobile home)

\begin{tabular}{|c|c|c|c|c|}
\hline EQD FMI & $\begin{array}{c}\text { Wall, } \\
\text { Ceiling }\end{array}$ & $\begin{array}{c}\text { Framing, } \\
\text { Insul. }\end{array}$ & $\begin{array}{l}\text { Bedding } \\
\text { Furn. }\end{array}$ & Othen \\
\hline $\begin{array}{l}\text { Fixed Wiring } \\
\text { Switch, Recep }\end{array}$ & 0.39 & & & \\
\hline $\begin{array}{l}\text { Cord, Plug } \\
\text { Bulb }\end{array}$ & & & & \\
\hline Other & & & & \\
\hline
\end{tabular}

HIGH RURAL

\begin{tabular}{|l|c|c|c|c|}
\hline EQP FMI & $\begin{array}{c}\text { Wall, } \\
\text { Ceiling }\end{array}$ & $\begin{array}{c}\text { Framing, } \\
\text { Insul. }\end{array}$ & $\begin{array}{c}\text { Bedding } \\
\text { Furn. }\end{array}$ & Other \\
\hline $\begin{array}{l}\text { Fixed Wiring } \\
\text { Switch, Recep }\end{array}$ & 0.24 & & & \\
\hline $\begin{array}{l}\text { Cord, Plug } \\
\text { Bulb }\end{array}$ & & & & \\
\hline Other & & & & \\
\hline
\end{tabular}

\begin{tabular}{|l|l|l|l|l|}
\hline EQP FMI & $\begin{array}{c}\text { Wall, } \\
\text { Ceiling }\end{array}$ & $\begin{array}{c}\text { Framing, } \\
\text { Insul. }\end{array}$ & $\begin{array}{c}\text { Bedding } \\
\text { Furn. }\end{array}$ & Other \\
\hline $\begin{array}{l}\text { Fixed Wiring } \\
\text { Switch, Recep. }\end{array}$ & & & & \\
\hline $\begin{array}{l}\text { Cord, Plug } \\
\text { Bulb }\end{array}$ & & & & 0.08 \\
\hline Other & & & & \\
\hline
\end{tabular}

HIGH NON-RURAI

\begin{tabular}{|l|c|c|c|c|}
\hline EQP FMI & $\begin{array}{c}\text { Wal1, } \\
\text { Ceiling }\end{array}$ & $\begin{array}{c}\text { Framing, } \\
\text { Insul. }\end{array}$ & $\begin{array}{c}\text { Bedding } \\
\text { Furn. }\end{array}$ & Other \\
\hline $\begin{array}{l}\text { Fixed Wiring } \\
\text { Switch, Recep }\end{array}$ & & & & \\
\hline $\begin{array}{l}\text { Cord, Plug } \\
\text { Bulb }\end{array}$ & & & & 0.03 \\
\hline Other & & & & \\
\hline
\end{tabular}

LOW NON-RURAL 
Table 27. Electrical distribution fire fatality rates - HIGH/LOW states (F1xed property use: other residential)

\begin{tabular}{|l|l|l|l|l|}
\hline EQP FMI & $\begin{array}{c}\text { Wa11, } \\
\text { Celling }\end{array}$ & $\begin{array}{c}\text { Fram1ng, } \\
\text { Insul. }\end{array}$ & $\begin{array}{c}\text { Bedd1ng } \\
\text { Furn. }\end{array}$ & Other \\
\hline $\begin{array}{l}\text { Fixed W1r1ng } \\
\text { Sw1tch, Recep }\end{array}$ & & & & \\
\hline $\begin{array}{l}\text { Cord, P1ug } \\
\text { Bulb }\end{array}$ & & & & \\
\hline Other & & & 0.13 & \\
\hline
\end{tabular}

HIGH RURAL

\begin{tabular}{|c|c|c|c|c|}
\hline EQP FMI & $\begin{array}{l}\text { Wall, } \\
\text { Celling }\end{array}$ & $\begin{array}{c}\text { Framing, } \\
\text { Insul. }\end{array}$ & $\begin{array}{l}\text { Bedding } \\
\text { Furn. }\end{array}$ & Other \\
\hline $\begin{array}{l}\text { Fixed Wiring } \\
\text { Switch, Recep }\end{array}$ & & 0.24 & & \\
\hline $\begin{array}{l}\text { Cord, Plug } \\
\text { Bulb }\end{array}$ & & : & & : \\
\hline Other & & . & . & . \\
\hline
\end{tabular}

LOW RURAL

\begin{tabular}{|l|l|c|c|c|}
\hline EQP FMI & $\begin{array}{c}\text { Wal1, } \\
\text { Celling }\end{array}$ & $\begin{array}{c}\text { Framing, } \\
\text { Insul. }\end{array}$ & $\begin{array}{c}\text { Bedd1ng } \\
\text { Furn. }\end{array}$ & Other \\
\hline $\begin{array}{l}\text { F1xed W1ring } \\
\text { Sw1tch, Recep. }\end{array}$ & & & & \\
\hline $\begin{array}{l}\text { Cord, Plug } \\
\text { Bulb }\end{array}$ & & & 0.08 & \\
\hline Other & & & & \\
\hline
\end{tabular}

HIGH NON-RURAL

\begin{tabular}{|l|l|l|l|l|}
\hline EQP FMI & $\begin{array}{c}\text { Wall, } \\
\text { Ceiling }\end{array}$ & $\begin{array}{c}\text { Framing, } \\
\text { Insul. }\end{array}$ & $\begin{array}{c}\text { Bedding } \\
\text { Furn. }\end{array}$ & Other \\
\hline $\begin{array}{l}\text { Fixed Wiring } \\
\text { Switch, Recep. }\end{array}$ & 0.03 & & $\vdots$ & \\
\hline $\begin{array}{l}\text { Cord, Plug } \\
\text { Bulb }\end{array}$ & & & 0.03 & 0.14 \\
\hline Other & 0.08 & 0.08 & & \\
\hline
\end{tabular}

LOW NON-RURAL

\title{
1. UNSAFE FIXED WIRING
}

\author{
RURAL Fatality Rate \\ NON-RURAL Fatality Rate \\ 1.42 fatalities/million \\ 0.32 fatalities/million
}

The two major components of the electrical distribution category of fire fatalities are related to fixed wiring, including switches, receptacles and junction boxes, and cords, plugs and light bulbs. Fixed wiring fires are usually within wall or ceiling concealed spaces and are often mechanical breakdowns resulting from defective installation or deterioration with age. Cord, plug and bulb type fires are more often directly associated with peoples actions (combustibles too close to bulbs, overloading or damage to extension cords etc.) and usually occur within the living space.

As the rates in tables 22-24 indicate, the RURAL fire fatality rate problem is primarily related to fixed wiring fires, with structural components (walls, ceilings, framing). The fatality rate for cord, plug, and bulb fires is similar for RURAL and NON-RURAL areas. From table 23 it is seen that fixed wiring fires in mobile homes are a significant component of the RURAL electrical distribution fire problem.

The following causal factors define this scenario:

Equipment: Fixed wiring, switch, receptacle Form of Material Ignited: Wall, ceiling, framing, insulation The fatality rates for this scenario are taken from tables 22, 23 and 24.

No information is available to breakdown RURAL fixed wiring fires by factors such as the proportion of installation defects or age deterioration. Tables 25-27 indicate, however, that fixed wiring fire fatalities are primarily a HIGH state problem, with the RURAL areas of HIGH states contributing the majority of the overall RURAL fatality rate in fixed wiring fires. 


\subsection{Open Flame Fire Scenarios}

The fatality rate in RURAI open flame fires (1.6) is higher than the rate for NON-RURAL fires (1.0) of this type. Table 28 gives the RURAL and NON-RURAL open flame fatality rates for ALL states for the factors found to be most significant for Open Flame Fires - Area of Origin (AO) and Form of Heat of Ignition (FHI). Table 29 provides the same breakdown for HIGH and LOW states also. Two scenarios were found to account for most of the RURAL/NON-RURAL discrepancy for open flame fires.

Table 28. Open flame fire fatality rates - ALL states

(Cause: Open flame)

\begin{tabular}{|l|c|c|c|c|}
\hline AO FHI & $\begin{array}{c}\text { Match } \\
\text { Lighter }\end{array}$ & Candle & $\begin{array}{c}\text { Open } \\
\text { Fire }\end{array}$ & Other \\
\hline $\begin{array}{l}\text { Living } \\
\text { Room }\end{array}$ & 0.08 & 0.05 & & 0.08 \\
\hline Bedroom & 0.08 & & & \\
\hline Outside & 0.17 & & 0.50 & \\
\hline Other & 0.08 & 0.08 & & \\
\hline
\end{tabular}

\begin{tabular}{|l|c|c|c|c|}
\hline A0 FHI & $\begin{array}{c}\text { Match } \\
\text { Lighter }\end{array}$ & Candle & $\begin{array}{c}\text { Open } \\
\text { Fire }\end{array}$ & Other \\
\hline $\begin{array}{l}\text { Living } \\
\text { Room }\end{array}$ & 0.14 & 0.16 & 0.02 \\
\hline Bedroom & 0.08 & 0.10 & & \\
\hline Outside & & & 0.12 & \\
\hline Other & 0.10 & & 0.02 & \\
\hline
\end{tabular}

ALL RURAL

ALL NON-RURAL

Table 29. Open flame fire fatality rates - HIGH/LOW states (Cause: Open flame)

\begin{tabular}{|l|c|c|c|c|}
\hline AO FHI & $\begin{array}{c}\text { Match } \\
\text { Lighter }\end{array}$ & Candle & $\begin{array}{c}\text { Open } \\
\text { Fire }\end{array}$ & Other \\
\hline $\begin{array}{l}\text { Living } \\
\text { Room }\end{array}$ & 0.13 & 0.78 & & 0.13 \\
\hline Bedroom & & & & \\
\hline Outside & 0.26 & & 0.52 & \\
\hline Other & & & & \\
\hline
\end{tabular}

\begin{tabular}{|l|c|l|l|l|}
\hline A0 FHI & $\begin{array}{c}\text { Match } \\
\text { Lighter }\end{array}$ & Candle & $\begin{array}{c}\text { Open } \\
\text { Fire }\end{array}$ & Other \\
\hline $\begin{array}{l}\text { Living } \\
\text { Room }\end{array}$ & 0.15 & 0.23 & & 0.08 \\
\hline Bedroom & 0.23 & 0.23 & & \\
\hline Outside & & & 0.23 & \\
\hline Other & & & & \\
\hline
\end{tabular}

HIGH RURAL

HIGH NON-RURAL

\begin{tabular}{|l|c|c|c|c|}
\hline AO FHI & $\begin{array}{c}\text { Match } \\
\text { Lighter }\end{array}$ & Candle & $\begin{array}{c}\text { Open } \\
\text { Fire }\end{array}$ & Other \\
\hline $\begin{array}{l}\text { Living } \\
\text { Room }\end{array}$ & & & & \\
\hline Bedroom & 0.24 & & & \\
\hline Outside & & & 0.47 & \\
\hline Other & 0.24 & 0.24 & & \\
\hline
\end{tabular}

\begin{tabular}{|l|c|c|c|l|}
\hline AOFHI & $\begin{array}{c}\text { Match } \\
\text { Lighter }\end{array}$ & Candle & $\begin{array}{c}\text { Open } \\
\text { Fire }\end{array}$ & Other \\
\hline $\begin{array}{l}\text { Living } \\
\text { Room }\end{array}$ & 0.14 & 0.14 & & \\
\hline Bedroom & 0.03 & 0.06 & & \\
\hline Outside & & & 0.08 & \\
\hline Other & 0.14 & & 0.03 & \\
\hline
\end{tabular}

LOW RURAL

LOW NON-RURAL 
RURAL Fatality Rate NON-RURAL Fatality Rate
0.67 fatalities/million

0.12 fatalities/million

The outside burning of brush, leaves, and trash, which is prohibited in many NON-RURAL areas, was a significant fatality cause in RURAL areas. Most of the victims were elderly males who ignited their clothing while burning or attempting to burn materials outside. The problem appears to exist in RURAL areas of both HIGH and LOW states.

The following causal factors define this scenario:

Area of Origin: Outside

Form of Heat: Open fire, match, lighter

\section{MISUSE OF CANDLES}

$\begin{array}{ll}\text { RURAL Fatality Rate } & 0.58 \text { fatalities/million } \\ \text { NON-RURAL Fatality Rate } & 0.26 \text { fatalities/million }\end{array}$

The use of candles for lighting accounted for a substantial portion of the RURAL/NON-RURAL open flame fatality rate discrepancy. Most of the victims were children. The main component is in the HIGH RURAL areas, with a lower, but similar rate in the remaining areas.

The following causal factors define this scenario:

Area of Origin: All interior

Form of Heat: Candle

\section{SELECTED GENERAL OBSERVATIONS}

While the causal factors involved in RURAL and NON-RURAL fire fatalities are of primary interest in this study, some general observations of other factors are worthy of note. Five factors (race, condition before injury, property type, smoke detectcr presence and fire department response) are discussed here, in the hope that they may provide some additional insight into possible reasons for the difference between RURAL and NON-RURAL fire fatality rates.

\subsection{RURAL and NON-RURAL Race Comparisons}

Information on population by race for each population interval in this study was not available. By making two assumptions, however, a population distribution for three intervals was calculated using Census statistics [5]. The intervals are RURAL (< 2500 population), URBAN (2500 - 50,000 population) and CENTRAL CITY (> 50,000 population). The assumptions made were:

- State population distributions by race remain the same as 1970 Census.

- RURAL/URBAN race distributions given nationally in the 1970 Census are a constant for each state and are unchanged from 1970.

Because of these necessary assumptions, and the simplified method used to calculate population by race, these distributions, and the subsequent fatality rates may be less reliable than those used in the remainder of this study. The general trends indicated are believed to be valid, however.

Figures 6 and 7 describe the population interval differences involving race that were noted in this study. Figure 6 indicates a total non-white fatality rate of slightly over twice the white fatality rate. This discrepancy is significantly more pronounced in the HIGH states. The USFA has reported similar findings [2]. 
FATALITY RATES BY RACE

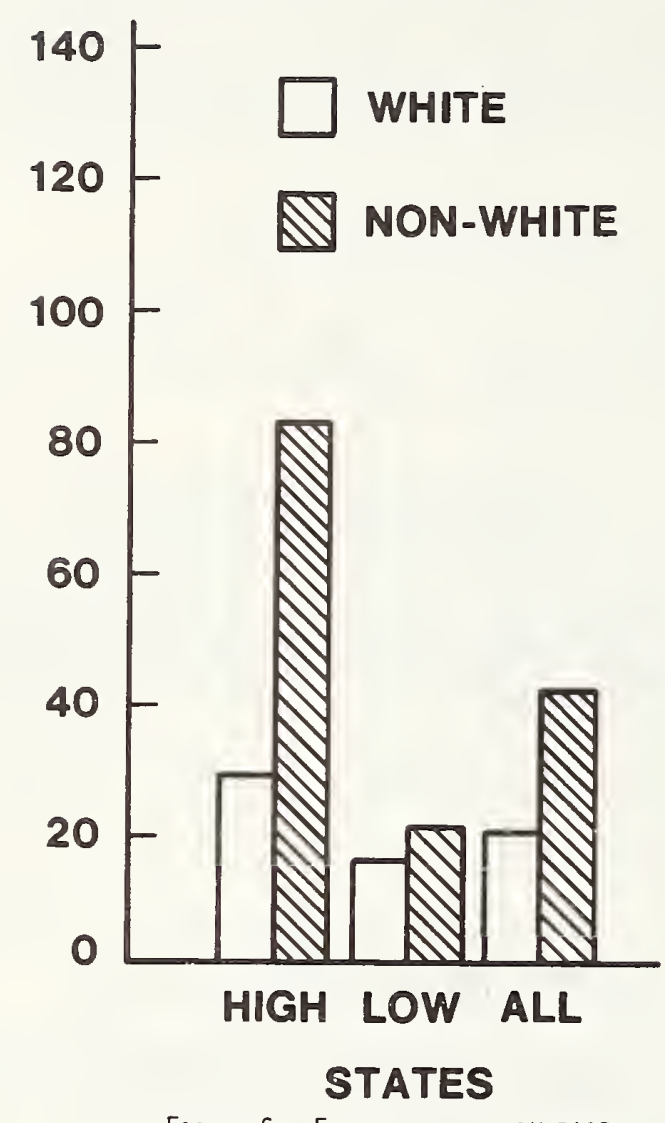

Figure 6. Fatality rates by race

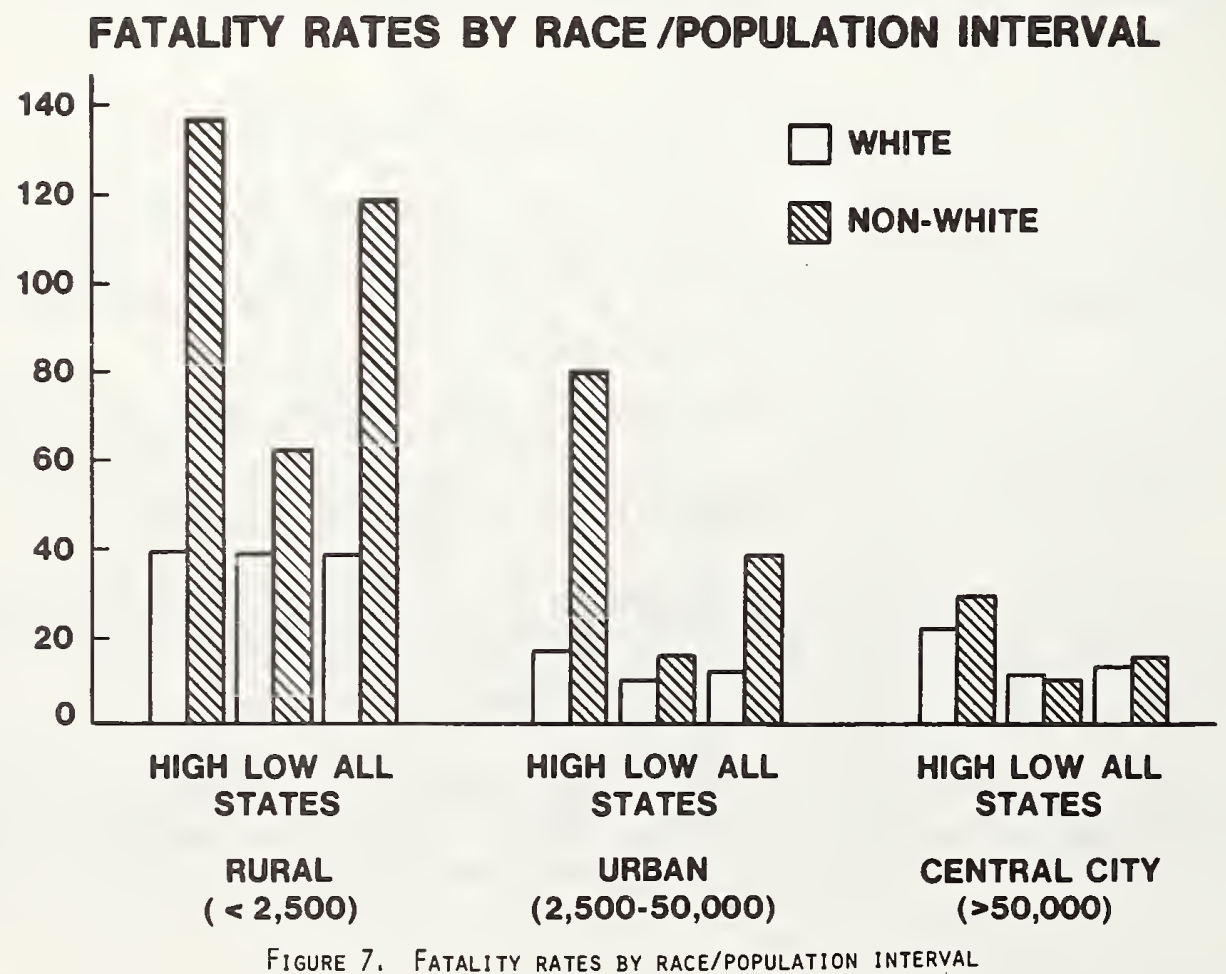


Figure 6 compares fatality rates by race for HIGH, LOW, and ALL states for the three population intervals. It appears that little difference in death rates between white and non-white exists in the CENTRAL CITIES, while RURAL and URBAN populations, exhibit an overall non-white rate of slightly over three times the white rate (ALL states).

It appears that the large differences between white and non-white death rates are in the RURAL and URBAN areas of the HIGH states, followed by the RURAL areas of the LOW states. These comparisons suggest that some characteristics of the high RURAL non-white fatality rate may be more pronounced in HIGH states, and may also exist in URBAN areas of HIGH states. Although it is beyond the scope of this study, more detailed examination of these rate differences may be useful in the future.

\subsection{Condition before Injury}

True fatality rates for certain classes of people (the elderly, for example) cannot be calculated based on population interval as the frequency of the class in the total population is not known. Some insight into the relative contribution of the fatalities in a given condition to the overall fatality rate for RURAL and NONRURAL areas can be gained however by comparing the percentage of fatalities in what has been coded here as "condition before injury" for all cases where the condition of the fatality before injury occurred is known. These tabulations are presented in table 30 for RURAL and NON-RURAL areas of ALL states, and are further broken down into HIGH and LOW states in table 31. The corresponding fatality rates for each condition before injury are also provided.

Table 30. Condition before injury - ALL states

\begin{tabular}{|c|c|c|c|}
\hline $\begin{array}{c}\text { Condition } \\
\text { Before } \\
\text { Injury }\end{array}$ & $\begin{array}{c}\text { Number } \\
\text { of } \\
\text { Fatalities }\end{array}$ & $\begin{array}{c}\text { Percent } \\
\text { of } \\
\text { Total }\end{array}$ & $\begin{array}{c}\text { Fatality } \\
\text { Rate }\end{array}$ \\
\hline Asleep & 160 & $33.3 \%$ & 13.46 \\
\hline $\begin{array}{c}\text { Bedridden, } \\
\text { Handicapped }\end{array}$ & 26 & $5.4 \%$ & 2.19 \\
\hline $\begin{array}{c}\text { Impaired by } \\
\text { Drugs, Alcoho1 }\end{array}$ & 100 & $20.8 \%$ & 8.41 \\
\hline $\begin{array}{c}\text { Too Young } \\
\text { To Act }\end{array}$ & 56 & $11.7 \%$ & 4.71 \\
\hline $\begin{array}{c}\text { Too 01d } \\
\text { To Act }\end{array}$ & 36 & $7.5 \%$ & 3.03 \\
\hline $\begin{array}{c}\text { Senile, Mentally } \\
\text { Handicapped }\end{array}$ & 861 & 7.91 \\
\hline $\begin{array}{c}\text { Awake, } \\
\text { Unimpaired }\end{array}$ & $1.7 \%$ & 0.67 \\
\hline
\end{tabular}

\begin{tabular}{|l|c|c|c|}
\hline $\begin{array}{c}\text { Condition } \\
\text { Before } \\
\text { Injury }\end{array}$ & $\begin{array}{c}\text { Number } \\
\text { of } \\
\text { Fatalities }\end{array}$ & $\begin{array}{c}\text { Percent } \\
\text { of } \\
\text { Total }\end{array}$ & $\begin{array}{c}\text { Fatality } \\
\text { Rate }\end{array}$ \\
\hline Asleep & 285 & $36.0 \%$ & 5.78 \\
\hline $\begin{array}{c}\text { Bedridden, } \\
\text { Handicapped }\end{array}$ & 54 & $6.8 \%$ & 1.09 \\
\hline $\begin{array}{c}\text { Impaired by } \\
\text { Drugs, Alcohol }\end{array}$ & 166 & $21.0 \%$ & 3.37 \\
\hline $\begin{array}{c}\text { Too Young } \\
\text { To Act }\end{array}$ & 136 & $17.2 \%$ & 2.76 \\
\hline $\begin{array}{c}\text { Too 01d } \\
\text { To Act }\end{array}$ & 64 & $8.1 \%$ & 1.30 \\
\hline $\begin{array}{c}\text { Senile, Mental1y } \\
\text { Handicapped }\end{array}$ & 21 & $2.7 \%$ & 0.43 \\
\hline $\begin{array}{c}\text { Awake, } \\
\text { Unimpaired }\end{array}$ & 65 & $8.2 \%$ & 1.32 \\
\hline
\end{tabular}

ALL RURAL

ALL NON-RURAL 
Table 31. Condition before injury - HIGH/LOW states

\begin{tabular}{|c|c|c|c|}
\hline $\begin{array}{c}\text { Condition } \\
\text { Before } \\
\text { Injury }\end{array}$ & $\begin{array}{c}\text { Number } \\
\text { of } \\
\text { Fatalities }\end{array}$ & $\begin{array}{c}\text { Percent } \\
\text { of } \\
\text { Total }\end{array}$ & $\begin{array}{c}\text { Fatality } \\
\text { Rate }\end{array}$ \\
\hline Asleep & 99 & $29.8 \%$ & 12.95 \\
\hline $\begin{array}{c}\text { Bedridden, } \\
\text { Handicapped }\end{array}$ & 20 & $6.0 \%$ & 2.62 \\
\hline $\begin{array}{c}\text { Impaired by } \\
\text { Drugs, Alcohol }\end{array}$ & 75 & $22.6 \%$ & 9.81 \\
\hline $\begin{array}{c}\text { Too Young } \\
\text { To Act }\end{array}$ & 70 & $21.1 \%$ & 9.16 \\
\hline $\begin{array}{c}\text { Too old } \\
\text { To Act }\end{array}$ & 18 & $5.4 \%$ & 2.35 \\
\hline $\begin{array}{c}\text { Senile, Mentally } \\
\text { Handicapped }\end{array}$ & $73.0 \%$ & 5.62 \\
\hline $\begin{array}{c}\text { Awake, } \\
\text { Unimpaired }\end{array}$ & $2.1 \%$ & 0.92 \\
\hline
\end{tabular}

HIGH RURAL

\begin{tabular}{|c|c|c|c|}
\hline $\begin{array}{c}\text { Condition } \\
\text { Before } \\
\text { Injury }\end{array}$ & $\begin{array}{c}\text { Number } \\
\text { of } \\
\text { Fatalities }\end{array}$ & $\begin{array}{c}\text { Percent } \\
\text { of } \\
\text { Total }\end{array}$ & $\begin{array}{c}\text { Fatality } \\
\text { Rate }\end{array}$ \\
\hline Asleep & 113 & $32.8 \%$ & 8.69 \\
\hline $\begin{array}{c}\text { Bedridden, } \\
\text { Handicapped }\end{array}$ & 27 & $7.8 \%$ & 2.08 \\
\hline $\begin{array}{c}\text { Impaired by } \\
\text { Drugs, Alcohol }\end{array}$ & 77 & $22.3 \%$ & 5.92 \\
\hline $\begin{array}{c}\text { Too Young } \\
\text { To Act }\end{array}$ & 62 & $18.0 \%$ & 4.77 \\
\hline $\begin{array}{c}\text { Too 0ld } \\
\text { To Act }\end{array}$ & 29 & $8.4 \%$ & 2.23 \\
\hline $\begin{array}{c}\text { Senile, Mentally } \\
\text { Handicapped }\end{array}$ & 13 & $3.8 \%$ & 1.00 \\
\hline $\begin{array}{c}\text { Awake, } \\
\text { Unimpaired }\end{array}$ & 24 & $7.0 \%$ & 1.85 \\
\hline
\end{tabular}

HIGH NON-RURAL

\begin{tabular}{|c|c|c|c|}
\hline $\begin{array}{c}\text { Condition } \\
\text { Before } \\
\text { Injury }\end{array}$ & $\begin{array}{c}\text { Number } \\
\text { of } \\
\text { Fatalities }\end{array}$ & $\begin{array}{c}\text { Percent } \\
\text { of } \\
\text { Total }\end{array}$ & $\begin{array}{c}\text { Fatality } \\
\text { Rate }\end{array}$ \\
\hline Asleep & 61 & $41.2 \%$ & 14.38 \\
\hline $\begin{array}{c}\text { Bedridden, } \\
\text { Handicapped }\end{array}$ & 19 & $12.8 \%$ & 4.48 \\
\hline $\begin{array}{c}\text { Impaired by } \\
\text { Drugs, Alcohol }\end{array}$ & 30 & $20.3 \%$ & 7.07 \\
\hline $\begin{array}{c}\text { Too Young } \\
\text { To Act }\end{array}$ & 13 & $8.8 \%$ & 3.06 \\
\hline $\begin{array}{c}\text { Too old } \\
\text { To Act }\end{array}$ & 18 & $12.2 \%$ & 4.24 \\
\hline $\begin{array}{c}\text { Senile, Mentally } \\
\text { Handicapped }\end{array}$ & 1 & $0.7 \%$ & 0.24 \\
\hline $\begin{array}{c}\text { Awake, } \\
\text { Unimpaired }\end{array}$ & & & 1.41 \\
\hline
\end{tabular}

\begin{tabular}{|c|c|c|c|}
\hline $\begin{array}{c}\text { Condition } \\
\text { Before } \\
\text { Injury }\end{array}$ & $\begin{array}{c}\text { Number } \\
\text { of } \\
\text { Asleep }\end{array}$ & $\begin{array}{c}\text { Percent } \\
\text { of } \\
\text { Total }\end{array}$ & $\begin{array}{c}\text { Fatality } \\
\text { Rate }\end{array}$ \\
\hline $\begin{array}{c}\text { Bedridden, } \\
\text { Handicapped }\end{array}$ & 27 & $38.6 \%$ & 4.74 \\
\hline $\begin{array}{c}\text { Impaired by } \\
\text { Drugs, Alcohol }\end{array}$ & 89 & $20.0 \%$ & 2.45 \\
\hline $\begin{array}{c}\text { Too Young } \\
\text { To Act }\end{array}$ & 74 & $16.6 \%$ & 2.04 \\
\hline $\begin{array}{c}\text { Too old } \\
\text { To Act }\end{array}$ & 35 & $7.8 \%$ & 0.06 \\
\hline $\begin{array}{c}\text { Senile, Mentally } \\
\text { Handicapped }\end{array}$ & 81 & $9.2 \%$ & 1.13 \\
\hline $\begin{array}{c}\text { Awake, } \\
\text { Unimpaired }\end{array}$ & $1.8 \%$ & 0.22 \\
\hline
\end{tabular}


As table 30 indicates, the distribution of fatalities by condition before injury is essentially similar in RURAL and NON-RURAL areas. A slightly higher proportion of fatalities in RURAL areas are classified as either "too young to act" or "too old to act". This discrepancy could be related to some characteristics of RURAL fires, but could also be due to a higher proportion of the young and the elderly in the RURAL population.

As table 31 indicates, the proportion of fatalities in the "too young to act" category is highest in the RURAL areas of both HIGH and LOW states, but the proportion of "too old to act" fatalities is significantly higher in the HIGH RURAL areas only.

Finally a relatively high proportion of "impaired by drugs, alcohol" fatalities appears to exist in both the RURAL and NON-RURAL areas of HIGH states. A comprehensive examination of the possible reasons for this apparent larger contribution of alcohol consumption to the fatalities in the HIGH states, including social, economic and legal (variability in state alcohol and drug laws for example) aspects might be helpful in addressing this problem.

\subsection{Property Type}

The distributions of residences by property type for RURAL and NON-RURAL areas are not available, and true fatality rates by property type therefore cannot be calculated. The comparison of relative fatality frequency by property type produces some interesting insights. Tables 32 and 33 give fatality frequencies and fatality rates for RURAL and NON-RURAL areas for ALL and HIGH/LOW states respectively.

As table 32 indicates, almost 25 percent of the RURAL fatalities occurred in mobile homes, as opposed to only 8 percent in mobile homes in NON-RURAL areas. Some of this discrepancy is almost certainly due to a larger proportion of mobile homes in RURAL areas. Census figures [9], for example, indicate that about 58 percent of all mobile homes are outside standard metropolitan statistical areas (SMSA) while only 38 percent of all single family dwellings are outside SMSA.'s. While a preponderance of apartments in NON-RURAL areas probably accounts for the higher frequency of apartment fire fatalities in NON-RURAL areas shown in table 32, since only about 10 percent of all multifamily units are located outside SMSA's it is not clear that the mobile home discrepancy can be completely accounted for by a larger proportion of mobile homes in the RURAL areas. Other factors, such as income level (mobile homes tend to be associated with lower income levels) and higher levels of risk in older mobile homes [10] may also be affecting this trend. As table 33 indicates, the high frequency of RURAL fire fatalities in mobile homes holds for both HIGH and LOW states.

Table 32. Property type - ALL states

\begin{tabular}{|l|c|c|c|}
\hline $\begin{array}{c}\text { Property } \\
\text { Type }\end{array}$ & $\begin{array}{c}\text { Number of } \\
\text { Fatalities }\end{array}$ & $\begin{array}{c}\text { Percent of } \\
\text { Total }\end{array}$ & $\begin{array}{c}\text { Fatality } \\
\text { Rate }\end{array}$ \\
\hline $\begin{array}{c}\text { One and } \\
\text { Two Family }\end{array}$ & 407 & $67.6 \%$ & 34.24 \\
\hline Apartment & 12 & $2.0 \%$ & 1.01 \\
\hline Mobile Home & 148 & $24.6 \%$ & 12.45 \\
\hline $\begin{array}{l}\text { Other } \\
\text { Residential }\end{array}$ & 35 & $5.9 \%$ & 2.94 \\
\hline
\end{tabular}

ALL RURAL

\begin{tabular}{|l|c|c|c|}
\hline $\begin{array}{c}\text { Property } \\
\text { Type }\end{array}$ & $\begin{array}{c}\text { Number of } \\
\text { Fatalities }\end{array}$ & $\begin{array}{c}\text { Percent of } \\
\text { Total }\end{array}$ & $\begin{array}{c}\text { Fatality } \\
\text { Rate }\end{array}$ \\
\hline $\begin{array}{l}\text { One and } \\
\text { Two Family }\end{array}$ & 595 & $59.6 \%$ & 12.06 \\
\hline Apartment & 234 & $23.4 \%$ & 4.74 \\
\hline Mobile Home & 80 & $8.0 \%$ & 1.62 \\
\hline $\begin{array}{l}\text { Other } \\
\text { Residential }\end{array}$ & 89 & $8.9 \%$ & 1.80 \\
\hline
\end{tabular}

ALL NON-RURAL 
Table 33. Property type - HIGH/LOW states

\begin{tabular}{|l|c|c|c|}
\hline $\begin{array}{c}\text { Property } \\
\text { Type }\end{array}$ & $\begin{array}{c}\text { Number of } \\
\text { Fatalities }\end{array}$ & $\begin{array}{c}\text { Percent of } \\
\text { Total }\end{array}$ & $\begin{array}{c}\text { Fatality } \\
\text { Rate }\end{array}$ \\
\hline $\begin{array}{c}\text { One and } \\
\text { Two Family }\end{array}$ & 287 & $68.2 \%$ & 37.54 \\
\hline Apartment & 7 & $1.7 \%$ & 0.92 \\
\hline Mobile Home & 110 & $26.1 \%$ & 14.39 \\
\hline $\begin{array}{l}\text { Other } \\
\text { Residential }\end{array}$ & 17 & $4.0 \%$ & 2.22 \\
\hline
\end{tabular}

HIGH RURAL

\begin{tabular}{|l|c|c|c|}
\hline $\begin{array}{c}\text { Property } \\
\text { Type }\end{array}$ & $\begin{array}{c}\text { Number of } \\
\text { Fatalities }\end{array}$ & $\begin{array}{c}\text { Percent of } \\
\text { Type }\end{array}$ & $\begin{array}{c}\text { Fatality } \\
\text { Rate }\end{array}$ \\
\hline $\begin{array}{c}\text { One and } \\
\text { Two Family }\end{array}$ & 120 & $66.3 \%$ & 28.29 \\
\hline Apartment & 5 & $2.8 \%$ & 1.18 \\
\hline Mobile Home & 38 & $21.0 \%$ & 8.96 \\
\hline $\begin{array}{l}\text { Other } \\
\text { Residential }\end{array}$ & 18 & $9.9 \%$ & 4.24 \\
\hline
\end{tabular}

LOW RURAL

\begin{tabular}{|l|c|c|c|}
\hline $\begin{array}{c}\text { Property } \\
\text { Type }\end{array}$ & $\begin{array}{c}\text { Number of } \\
\text { Fatalities }\end{array}$ & $\begin{array}{c}\text { Percent of } \\
\text { Total }\end{array}$ & $\begin{array}{c}\text { Fatality } \\
\text { Rate }\end{array}$ \\
\hline $\begin{array}{c}\text { One and } \\
\text { Two Family }\end{array}$ & 305 & $73.5 \%$ & 23.45 \\
\hline Apartment & 62 & $14.9 \%$ & 4.77 \\
\hline Mobile Home & 36 & $8.6 \%$ & 2.77 \\
\hline $\begin{array}{l}\text { Other } \\
\text { Residential }\end{array}$ & 12 & $2.9 \%$ & 0.92 \\
\hline
\end{tabular}

HIGH NON-RURAL

\begin{tabular}{|l|c|c|c|}
\hline $\begin{array}{c}\text { Property } \\
\text { Type }\end{array}$ & $\begin{array}{c}\text { Number of } \\
\text { Fatalities }\end{array}$ & $\begin{array}{c}\text { Percent of } \\
\text { Type }\end{array}$ & $\begin{array}{c}\text { Fatality } \\
\text { Rate }\end{array}$ \\
\hline $\begin{array}{c}\text { One and } \\
\text { Two Family }\end{array}$ & 290 & $49.7 \%$ & 7.98 \\
\hline Apartment & 172 & $29.5 \%$ & 4.74 \\
\hline Mobile Home & 44 & $7.6 \%$ & 1.21 \\
\hline $\begin{array}{c}\text { Other } \\
\text { Residential }\end{array}$ & 77 & $13.3 \%$ & 2.12 \\
\hline
\end{tabular}

LOW NON-RURAL

\section{4 Smoke Detector Presence}

Tables 34 and 35 tabulate smoke detector presence and operation for RURAL and NON-RURAL areas of ALI and HIGH/LOW states respectively. The proportion of smoke detector protected homes in the studied states is not known, and true fatality rates classified by smoke detector presence therefore cannot be calculated. About 6 percent of all fatalities occurred in homes with smoke detectors in all of the areas studied. RURAL areas, and the HIGH states show slightly higher fatality rates for smoke detectors present than NON-RURAL areas of LOW states. This may be due to fewer smoke detectors in these areas. The higher incidence of clothing ignitions in these areas may also contribute to this higher rate. The fatalities are too few, and the proportion of smoke detector protected homes in the study area is too uncertain, to attempt to draw conclusions regarding smoke detector performance here however.

Table 34. Smoke detector presence - ALL states

\begin{tabular}{|l|c|c|c|}
\hline \multicolumn{1}{|c|}{$\begin{array}{c}\text { Smoke } \\
\text { Detector }\end{array}$} & $\begin{array}{c}\text { Number of } \\
\text { Fatalities }\end{array}$ & $\begin{array}{c}\text { Percent of } \\
\text { Total }\end{array}$ & $\begin{array}{c}\text { Fatality } \\
\text { Rate }\end{array}$ \\
\hline $\begin{array}{l}\text { Present \& } \\
\text { Operated }\end{array}$ & 4 & $1.0 \%$ & 0.34 \\
\hline $\begin{array}{l}\text { Present \& Did } \\
\text { Not Operate }\end{array}$ & 5 & $1.3 \%$ & 0.42 \\
\hline $\begin{array}{l}\text { Present \& Oper- } \\
\text { ation Unknown }\end{array}$ & 14 & $3.6 \%$ & 1.18 \\
\hline None Present & 361 & $94.0 \%$ & 30.37 \\
\hline
\end{tabular}

\begin{tabular}{|l|c|c|c|}
\hline $\begin{array}{c}\text { Smoke } \\
\text { Detector }\end{array}$ & $\begin{array}{c}\text { Number of } \\
\text { Fatalities }\end{array}$ & $\begin{array}{c}\text { Percent of } \\
\text { Total }\end{array}$ & $\begin{array}{c}\text { Fatality } \\
\text { Rate }\end{array}$ \\
\hline $\begin{array}{l}\text { Present \& } \\
\text { Operated }\end{array}$ & 8 & $1.5 \%$ & 0.16 \\
\hline $\begin{array}{l}\text { Present \& Did } \\
\text { Not Operate }\end{array}$ & 17 & $3.2 \%$ & 0.34 \\
\hline $\begin{array}{l}\text { Present \& Oper- } \\
\text { ation Unknown }\end{array}$ & 6 & $1.1 \%$ & 0.12 \\
\hline None Present & 496 & $94.1 \%$ & 10.05 \\
\hline
\end{tabular}


Table 35. Smoke detector presence - HIGH/LOW states

\begin{tabular}{|l|c|c|c|}
\hline $\begin{array}{c}\text { Smoke } \\
\text { Detector }\end{array}$ & $\begin{array}{c}\text { Number of } \\
\text { Fatalities }\end{array}$ & $\begin{array}{c}\text { Percent of } \\
\text { Total }\end{array}$ & $\begin{array}{c}\text { Fatality } \\
\text { Rate }\end{array}$ \\
\hline $\begin{array}{l}\text { Present \& } \\
\text { Pperated }\end{array}$ & - & $0.0 \%$ & - \\
\hline $\begin{array}{l}\text { Present \& Did } \\
\text { Not Operate }\end{array}$ & 5 & $1.7 \%$ & 0.65 \\
\hline $\begin{array}{l}\text { Present \& Oper- } \\
\text { ation Unknown }\end{array}$ & 11 & $3.7 \%$ & 1.44 \\
\hline None Present & 284 & $94.7 \%$ & 37.15 \\
\hline
\end{tabular}

HIGH RURAL

\begin{tabular}{|l|c|c|c|}
\hline $\begin{array}{c}\text { Smoke } \\
\text { Detector }\end{array}$ & $\begin{array}{c}\text { Number of } \\
\text { Fatalities }\end{array}$ & $\begin{array}{c}\text { Percent of } \\
\text { Total }\end{array}$ & $\begin{array}{c}\text { Fatality } \\
\text { Rate }\end{array}$ \\
\hline $\begin{array}{l}\text { Present \& } \\
\text { Operated }\end{array}$ & 4 & $4.8 \%$ & 0.94 \\
\hline $\begin{array}{l}\text { Present \& Did } \\
\text { Not Operate }\end{array}$ & - & $0.0 \%$ & - \\
\hline $\begin{array}{l}\text { Present \& Oper- } \\
\text { ation Unknown }\end{array}$ & 3 & $3.6 \%$ & 0.71 \\
\hline None Present & 77 & $91.7 \%$ & 18.15 \\
\hline
\end{tabular}

LOW RURAL

\begin{tabular}{|l|c|c|c|}
\hline $\begin{array}{c}\text { Smoke } \\
\text { Detector }\end{array}$ & $\begin{array}{c}\text { Number of } \\
\text { Fatalities }\end{array}$ & $\begin{array}{c}\text { Percent of } \\
\text { Total }\end{array}$ & $\begin{array}{c}\text { Fatality } \\
\text { Rate }\end{array}$ \\
\hline $\begin{array}{l}\text { Present \& } \\
\text { Operated }\end{array}$ & 4 & $1.3 \%$ & 0.31 \\
\hline $\begin{array}{l}\text { Present \& Did } \\
\text { Not Operate }\end{array}$ & 13 & $4.3 \%$ & 1.00 \\
\hline $\begin{array}{l}\text { Present \& Oper- } \\
\text { ation Unknown }\end{array}$ & - & $0.0 \%$ & - \\
\hline None Present & 287 & $94.4 \%$ & 22.06 \\
\hline
\end{tabular}

HIGH NON-RURAL

\begin{tabular}{|l|c|c|c|}
\hline $\begin{array}{c}\text { Smoke } \\
\text { Detector }\end{array}$ & $\begin{array}{c}\text { Number of } \\
\text { Fatalities }\end{array}$ & $\begin{array}{c}\text { Percent of } \\
\text { Total }\end{array}$ & $\begin{array}{c}\text { Fatality } \\
\text { Rate }\end{array}$ \\
\hline $\begin{array}{l}\text { Present \& } \\
\text { Pperated }\end{array}$ & 4 & $1.8 \%$ & 0.11 \\
\hline $\begin{array}{l}\text { Present \& Did } \\
\text { Not Operate }\end{array}$ & 4 & $1.8 \%$ & 0.11 \\
\hline $\begin{array}{l}\text { Present \& Oper- } \\
\text { ation Unknown }\end{array}$ & 6 & $2.7 \%$ & 0.17 \\
\hline None Present & 209 & $93.7 \%$ & 5.75 \\
\hline
\end{tabular}

LOW NON-RURAL

\subsection{Fire Department Response}

of the 1606 civilian residential fire fatalities, 1430 (89.0\%) were responded to by a fire department. There was no fire department response in 91 ( $5.7 \%$ ) of the cases, and response was unknown in 85 (5.3\%) of the cases. Adjusting for unknown response gives an estimate that $6.0 \%$ of fatalities are not responded to by $f i r e$ departments, and are therefore not likely to be reported to fire departments. These unreported fire fatalities were located through death certificates, and information on them was obtained through contact with local authorities.

As table 36 illustrates, unreported fire fatalities are most common in the HIGH states, particularly in the RURAL areas of those states. Almost 15 percent of the fatalities in HIGH RURAL areas are unreported. Of the 91 unreported fatalities in the data base, 80 (88\%) are in the HIGH states. A total of 60 (66\%) are in the RURAL areas of those HIGH states. The dramatic of unreported fire fatalities in RURAL areas of the HIGH states is further illustrated by comparison of fatality rates in table 36 . 
Table 36. Reported and unreported fatalities - HIGH/LOW states

\begin{tabular}{|l|c|c|c|}
\hline $\begin{array}{l}\text { Fire Dept. } \\
\text { Response }\end{array}$ & $\begin{array}{c}\text { Number of } \\
\text { Fatalities }\end{array}$ & $\begin{array}{c}\text { Percent of } \\
\text { Total }\end{array}$ & $\begin{array}{c}\text { Fatality } \\
\text { Rate }\end{array}$ \\
\hline Unreported & 60 & 14.2 & 7.9 \\
\hline Reported & 346 & 82.2 & 45.3 \\
\hline Unknown & 15 & 3.6 & - \\
\hline
\end{tabular}

HIGH RURAL

\begin{tabular}{|l|c|c|c|}
\hline $\begin{array}{l}\text { Fire Dept. } \\
\text { Response }\end{array}$ & $\begin{array}{c}\text { Number of } \\
\text { Fatalities }\end{array}$ & $\begin{array}{c}\text { Percent of } \\
\text { Total }\end{array}$ & $\begin{array}{c}\text { Fatality } \\
\text { Rate }\end{array}$ \\
\hline Unreported & 20 & 4.8 & 1.5 \\
\hline Reported & 392 & 94.5 & 30.1 \\
\hline Unknown & 3 & 0.7 & - \\
\hline
\end{tabular}

HIGH NON-RURAL

\begin{tabular}{|l|c|c|c|}
\hline $\begin{array}{l}\text { Fire Dept } \\
\text { Response }\end{array}$ & $\begin{array}{c}\text { Number of } \\
\text { Fatalities }\end{array}$ & $\begin{array}{c}\text { Percent of } \\
\text { Total }\end{array}$ & $\begin{array}{c}\text { Fatality } \\
\text { Rate }\end{array}$ \\
\hline Unreported & 7 & 3.9 & 1.7 \\
\hline Reported & 149 & 82.3 & 35.1 \\
\hline Unknown & 25 & 13.8 & - \\
\hline
\end{tabular}

LOW RURAL

\begin{tabular}{|l|c|c|c|}
\hline $\begin{array}{l}\text { Fire Dept. } \\
\text { Response }\end{array}$ & $\begin{array}{c}\text { Number of } \\
\text { Fatalities }\end{array}$ & $\begin{array}{c}\text { Percent of } \\
\text { Total }\end{array}$ & $\begin{array}{c}\text { Fatality } \\
\text { Rate }\end{array}$ \\
\hline Unreported & 4 & 0.7 & 0.1 \\
\hline Reported & 540 & 92.6 & 14.9 \\
\hline Unknown & 39 & 6.7 & - \\
\hline
\end{tabular}

LOW NON-RURAL

Unreported fire fatalities in each of the HIGH states are tabulated in table 37. Oklahoma shows only one unreported fatality, and has none in RURAL areas which is significantly lower than the other HIGH states. The remaining five states exhibit similar trends in unreported fire fatalities, except that Arkansas has a significantly higher proportion of unreported fatalities than the other states.

Table 37. Unreported fatalities - HIGH states

\begin{tabular}{|c|c|c|c|c|c|c|}
\hline State & $\begin{array}{c}\text { Number } \\
\text { Unreported }\end{array}$ & $\begin{array}{l}\text { NAL } \\
\text { Reported }\end{array}$ & $\begin{array}{c}\text { Percent } \\
\text { Unreported }\end{array}$ & $\begin{array}{c}\text { Number } \\
\text { Unreported }\end{array}$ & $\begin{array}{l}\text { NON-RUR } \\
\text { Number } \\
\text { Reported }\end{array}$ & $\begin{array}{c}\text { Percent } \\
\text { Unreported }\end{array}$ \\
\hline Alabama & 11 & 73 & $13.1 \%$ & 0 & 79 & - \\
\hline Arkansas & 13 & 23 & $36.1 \%$ & 6 & 35 & $14.6 \%$ \\
\hline Georgia & 14 & 85 & $14.1 \%$ & 9 & 105 & $7.9 \%$ \\
\hline Mississippi & 8 & 67 & $10.7 \%$ & 3 & 33 & $8.3 \%$ \\
\hline Oklahoma & 0 & 29 & - & 1 & 59 & $1.7 \%$ \\
\hline Tennessee & 14 & 69 & $16.9 \%$ & 1 & 81 & $1.2 \%$ \\
\hline
\end{tabular}

Unreported fatalities are tabulated by cause code in table 38 . As noted earlier, unreported fatalities are a substantial contributer to the overall fatality rate in HIGH RURAL areas only. Of the known causes, unreported fatalities involving heating predominate. Many are undoubtedly clothing ignitions, one of the most significant scenarios in RURAL areas, which are more likely to be unreported than other scenarios. (A total of 38 fatalities, or 39.2 percent of the 97 clothing ignitions in the incident data base were not reported to the fire department.) 
Table 38. Unreported fatalities by cause

\begin{tabular}{|l|c|c|c|}
\hline Cause & $\begin{array}{c}\text { Number of } \\
\text { Fatalities }\end{array}$ & $\begin{array}{c}\text { Percent of } \\
\text { Cause Total }\end{array}$ & $\begin{array}{c}\text { Fatality } \\
\text { Rate }\end{array}$ \\
\hline Heating & 23 & $19.8 \%$ & 3.0 \\
\hline Cooking & 5 & $14.7 \%$ & 0.7 \\
\hline Smoking & 5 & $9.8 \%$ & 0.7 \\
\hline Open Flame & 4 & $28.6 \%$ & 0.5 \\
\hline Unknown & 22 & $15.4 \%$ & 2.9 \\
\hline All Other & 1 & $2.1 \%$ & 0.1 \\
\hline
\end{tabular}

\begin{tabular}{|l|c|c|c|}
\hline Cause & $\begin{array}{c}\text { Number of } \\
\text { Fatalities }\end{array}$ & $\begin{array}{c}\text { Percent of } \\
\text { Cause Total }\end{array}$ & $\begin{array}{c}\text { Fatality } \\
\text { Rate }\end{array}$ \\
\hline Heating & 7 & $8.0 \%$ & 0.5 \\
\hline Cooking & 2 & $7.4 \%$ & 0.2 \\
\hline Smoking & 1 & $0.9 \%$ & 0.1 \\
\hline Open Flame & 4 & $21.1 \%$ & 0.3 \\
\hline Unknown & 5 & $5.7 \%$ & 0.4 \\
\hline All Other & 1 & $1.3 \%$ & 0.1 \\
\hline
\end{tabular}

HIGH RURAL

HIGH NON-RURAL

\begin{tabular}{|l|c|c|c|}
\hline Cause & $\begin{array}{c}\text { Number of } \\
\text { Fatalities }\end{array}$ & $\begin{array}{c}\text { Percent of } \\
\text { Cause Total }\end{array}$ & $\begin{array}{c}\text { Fatality } \\
\text { Rate }\end{array}$ \\
\hline Heating & 1 & $2.4 \%$ & 0.2 \\
\hline Cooking & 3 & $18.8 \%$ & 0.7 \\
\hline Smoking & 1 & $2.8 \%$ & 0.2 \\
\hline Open Flame & 1 & $25.0 \%$ & 0.2 \\
\hline Unknown & 1 & $3.3 \%$ & 0.2 \\
\hline All Other & 0 & - & - \\
\hline
\end{tabular}

\begin{tabular}{|l|c|c|c|}
\hline \multicolumn{1}{|c|}{ Cause } & $\begin{array}{c}\text { Number of } \\
\text { Fatalities }\end{array}$ & $\begin{array}{c}\text { Percent of } \\
\text { Cause Total }\end{array}$ & $\begin{array}{c}\text { Fatality } \\
\text { Rate }\end{array}$ \\
\hline Heating & 0 & - & - \\
\hline Cooking & 1 & $2.5 \%$ & 0.03 \\
\hline Smoking & 2 & $1.1 \%$ & 0.06 \\
\hline Open Flame & 0 & - & - \\
\hline Unknown & 1 & $1.3 \%$ & 0.03 \\
\hline All Other & 0 & - & - \\
\hline
\end{tabular}

LOW RURAL

LOW NON-RURAL

The unreported fire fatalities where form of heat of ignition is known are tabulated in table 39. Solid fueled equipment ( 31 percent) and gas fueled equipment (28 percent) make up the majority of the unreported total. They are largely clothing ignitions of people sitting too close to gas space heaters and wood stoves. In terms of proportion of unreported fatalities to reported for each form of heat, open fires (38.5 percent unreported) lead. This is expected, as many open fires (i.e. burning of leaves, brush, etc. outside) do not result in an uncontrolled fire which would necessitate calling the fire department. The high (16.5 percent) proportion of solid fueled equipment fatalities which are unreported include a preponderance of wood heat in RURAL areas not protected by local fire departments, or the use of wood stoves. as the sole source of heat by the elderly RURAL population, who may sit too close to the wood stove to keep warm. 
Table 39. Unreported fatalities by form of heat of ignition

\begin{tabular}{|lccc|}
\hline $\begin{array}{c}\text { Form Heat } \\
\text { of Ignition }\end{array}$ & $\begin{array}{c}\text { Number of Unreported } \\
\text { Fatalities }\end{array}$ & $\begin{array}{c}\text { Percent of Unreported } \\
\text { Total }\end{array}$ & $\begin{array}{c}\text { Proportion of Unreported } \\
\text { to Reported }\end{array}$ \\
\hline Gas Fueled Equipment & 16 & $28 \%$ & $9.5 \%$ \\
Liquid Fueled Equipment & 3 & $5 \%$ & $0.1 \%$ \\
Solid Fueled Equipment & 18 & $31 \%$ & $16.5 \%$ \\
Electrical & 2 & $3 \%$ & $0.8 \%$ \\
Smoking Materials & 9 & $15 \%$ & $2.7 \%$ \\
Open Fire & 5 & $9 \%$ & $38.5 \%$ \\
Other Open Flame & 5 & $9 \%$ & $7.2 \%$ \\
Other & 0 & - & - \\
\multicolumn{1}{|c|}{ Total } & 58 & & \\
\hline
\end{tabular}

\section{CONCLUSIONS}

In the course of this study information was obtained on 1606 civilian residential fire fatalities which occurred in 12 states over a period of one year. Six of the states were characterized by much higher than average annual fire fatality rates, while the rates in the remainder were much lower than the national average. The primary thrust of this study was to examine the differences in RURAL $(<2500$ population) and NON-RURAL (> 2500 population) areas with respect to fire causal factors. Some analysis of causal factors in the HIGH death rate states versus the LOW death rate states was also accomplished.

When the fatality rates (deaths per million population) calculated in this study were aggregated by population interval they were found to be consistent with previously reported analyses of this type, with RURAL areas having significantly higher fire fatality rates than any other population and with an overall RURAI fatality rate of $2-1 / 2$ times the NON-RURAL rate. The basic causal factors (Area of Origin, Equipment Involved in Ignition, Form of Heat of Ignition, Type of Material Ignited, Form of Material Ignited and Ignition Factor) were compared for RURAL and NON-RURAL areas using fatality rates as the means of comparison.

Of the known fire causes it was found that heating fires produced by far the largest RURAL fire fatality rate. It was also found that, while the RURAL fatality rates were higher than the NON-RURAL rates for all causes except Incendiary/Suspicious fires, the greatest contribution to the overall difference between the RURAL and NON-RURAL fatality rates is made by heating fires.

The most significant basic fire causes were examined in detail for RURAL and NON-RURAL fires by developing fire cause scenarios consisting of more detailed descriptions of the basic fire causes. For each basic fire cause the scenarios contributing the most toward the RURAL/NON-RURAL fatality rate discrepancy for that cause were identified and described.

The improper installation or misuse of solid fueled heating equipment was found to be the most significant RURAL fire problem. The misuse of solid fueled equipment included placing combustibles too close to the heat, ignition of clothing on people and using flammable liquids to kindle. A significant problem with solid fueled heating equipment was also noted in NON-RURAL areas of the HIGH states.

Other significant RURAL fire cause scenarios include unattended cooking, smoking, unsafe fixed wiring, outside burning, and misuse of gas fueled local heating equipment and portable space heaters. 
Examination of non-causal factors revealed several fatality rate characteristics of potential significance. Characterization of fatality rates by race (white and non-white) indicated a substantially higher non-white fatality rate in all areas except for central cities ( $>50,000$ population). The highest non-white fatality rates were in the RURAL areas of the HIGH states. Fires in mobile homes contributed considerably more to the overall fatality rates in RURAL areas than in NON-RURAL areas, and a higher proportion of elderly people appear to be at risk in RURAL areas.

While this study addresses primarily the physical causes of fire fatalities, it appears obvious that socio-economic factors play an important underlying role in both RURAI and HIGH state fatality rates. To increase our understanding of the relationships between socio-economic conditions and fire causes, and to better identify possible fire fatality reduction strategies additional study of both should be undertaken. Toward this end additional data on fire fatalities in several states are currently being collected, and will subsequently be analyzed in an attempt to better characterize RURAL fire problems.

\section{ACKNOWLEDGEMENT}

The authors gratefully acknowledge the contributions to the study of the state Fire Marshal's offices, the fire services, and other involved individuals in the states of Alabama, Arkansas, California, Connecticut, Delaware, Florida, Georgia, Mississippi, Oklahoma, Tennessee, Utah, and Wisconsin.

\section{REFERENCES}

[1] Fire in the United States: Deaths, Injuries, Dollar Loss, and Incidents at the National, State and Local Levels. National Fire Prevention and Control Administration, Department of Commerce, (U.S.), December 1978.

[2] Highlights of Fire in the United States, Second Edition, U.S. Fire Administration, Federal Emergency Management Administration (U.S.), April 1981.

[3] Uniform Coding for Fire Protection 1976, NFPA No. 901, National Fire Protection Assn., Boston, MA 02210, 1976.

[4] 1980 Commercial Atlas and Marketing Guide, lloth Edition, Rand McNally and Company, 1980.

[5] Karter, Michael J., Jr., Fire Loss in the United States During 1979, Fire Journal, Vol. 74, No. 5, pp. 52-65, Sept. 1980.

[6] Statistical Abstract of the United States: 1979, U.S. Bureau of the Census, 1979.

[7] Population and Land Area of Urbanized Areas for the United States: 1970 and 1960 , U.S. Department of Commerce, Bureau of the Census, Supplementary Report PC(SI)-108, April 1979.

[8] Population Estimates and Projections - 1977, U.S. Department of Commerce, Bureau of the Census, Series P-25, November 1979.

[9] Statistical Abstract of the United States: 1980, U.S. Bureau of the Census, 1980 .

[10] Wilson, Rexford and Gomberg, Alan, The Occupant Risk Method, Firepro Inc., Wellesley Hills, MA, 1978. 
APPENDIX A

CODING STRUCTURES

CODING FOR FIRE FATAIITY WORKSHEETS

ID NUMBER ( 5 digits)

- first two digits used for NCHS state code (attached)

MULTIPLE FATALITY FIRE NUMBER (2 digits)

- number each multiple fatality fire under ID number 00 for single fatality fire

\section{BLOCK A}

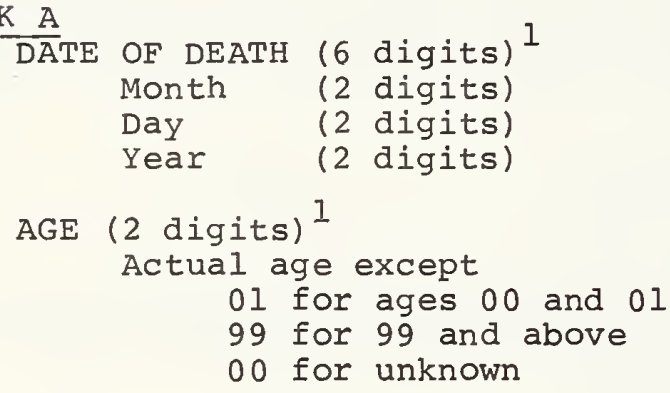

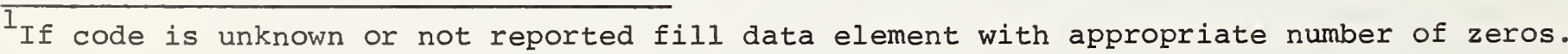




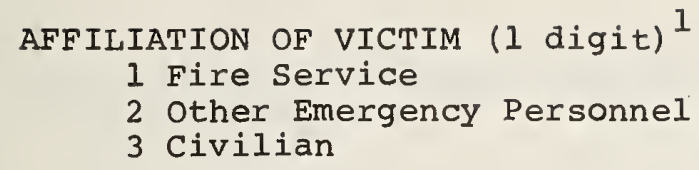

BLOCK D

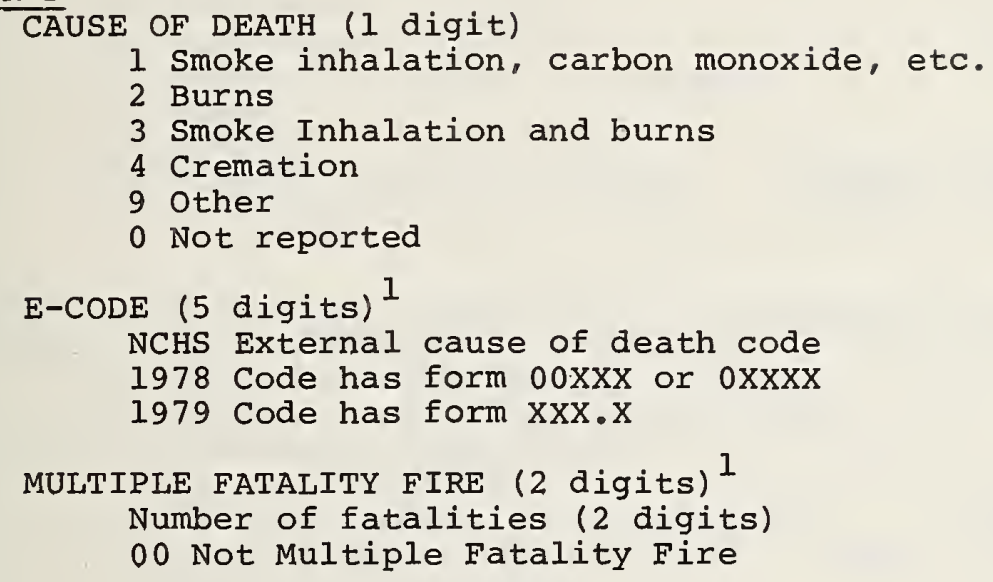

BLOCK E

ADDRESS (13 digits) 1

Street Address - Omit

City/Town ( 5 digit census code)

Zip Code (5 digits)

County ( 3 digit census code)

\section{BLOCK F}

WAS FIRE DEPARTMENT CALLED? IF YES, TYPE OF FIRE DEPARTMENT ( 1 digit)

0 Not caIled

1 Yes, all paid

2 Yes, part paid

3 Yes, all volunteer

8 Response unknown

9 Yes, type unknown

RUN TIME (In minutes -2 digits) $^{1}$

00 Unknown

BLOCK G

$$
\begin{aligned}
& \text { POPULATION OF PLACE (2 digits) } \\
& 01 \text { Under 2,500 } \\
& 022,500-5,000 \\
& 035,000-10,000 \\
& 0410,000-25,000 \\
& 0525,000-50,000 \\
& 0650,000-100,000 \\
& 07100,000-250,000 \\
& 08250,000-500,000 \\
& 09500,000-1,000,000 \\
& 10 \text { Over 1,000,000 } \\
& 00 \text { Not reported }
\end{aligned}
$$


PROPERTY TYPE (2 digits)

41 One/two family

42 Apartment

47 Mobile home

44 Hotel

04 Other residential

01 Public assembly

02 Educational

03 Institutional

05 Store, office

06 Industry, utility, defense

08 Storage

07 Manufacturing

"Other" is coded with appropriate 2 digit NFIRS code ${ }^{2}$ except 09 Vehicle fire

45 Travel trailer

BLOCK I

ORIGIN OF FIRE (2 digits)

14 Living room/family room

21 Bedroom

23 Dining room

24 Kitchen

25 Bathroom

42 Closet

47 Garage/carport

62 Heating equipment room/water heater area

75 Concealed wall space

00 Undetermined/not reported

"Not Classified Above" is coded with appropriate NFIRS ${ }^{2}$ code

BLOCK J

EQUIPMENT INVOLVED (2 digits)

11 Central heating unit

13 Stationary local heating unit

14 Fireplace

15 Portable local heating unit

01 Chimney

02 Cooking equipment

03 Fixed wiring

04 Switch, receptacle, outlet

05 Cord, plug

06 Television, radio, phonograph

08 No equipment involved

00 Undetermined/not reported

"Not Classified Above" coded with appropriate NFIRS code ${ }^{2}$

BLOCK K

FORM OF HEAT IGNITION (2 digits)

01 Gas fueled equipment

02 Liquid fueled equipment

03 Solid fueled equipment

04 short circuit

31 Cigarette

44 Candle

45 Match

46 Lighter

05 Properly operating electrical equipment

06 Improperly operating electrical equipment

00 Undetermined/not reported

"Not Classified Above" coded with appropriate NFIRS code ${ }^{2}$ 
BLOCK L

TYPE OF MATERIAL IGNITED (2 digits)

01 Natural gas

02 LP gas

03 Gasoline

04 Cooking oil, grease

05 other food

06 Sawn wood, framing

07 Hardwood, plywood, fiberboard

08 Paper

09 Fabric

00 Undetermined/not reported

"Not Classified Above" coded with appropriate NFIRS code ${ }^{2}$

\section{BLOCK M}

FORM OF MATERIAL IGNITED (2 digits)

01 Interior wall covering, surface

02 Thermal, acoustical insulation

21 Upholstered furniture

03 Mattress/bedding

34 Wearing apparel not on a person

35 Wearing apparel on a person

44 Magazine, newspaper

04 Rubbish, trash waste

05 Cooking materials

00 Undetermined/not reported

"Not Classified Above" coded with appropriate NFIRS code ${ }^{2}$

\section{BLOCK N}

IGNITION FACOTR ( 2 digits)

01 Incendiary/suspicious

31 Abandoned, discarded material (cigarette)

33 Falling asleep

36 Children playing

41 Fuel spilled, released accidentally

46 Combustible too close to heat

51 Part failure, leak, break

54 Short circuit, ground fault

56 Lack of mainenance, worn out

63 Installed too close to combustibles

73 Unattended

"Not Classified Above" coded with appropriate NFIRS code except ${ }^{2}$

08 Murder

Q9 Suicide

\section{BLOCK O}

LOCATION AT TIME OF IGNITION ( 1 digit)

1 Intimately involved with ignition

2 In the room or space of fire origin

4 In same building as origin of fire

5 Outside of building of fire origin

0 Undetermined/not reported

9 Not classified above 


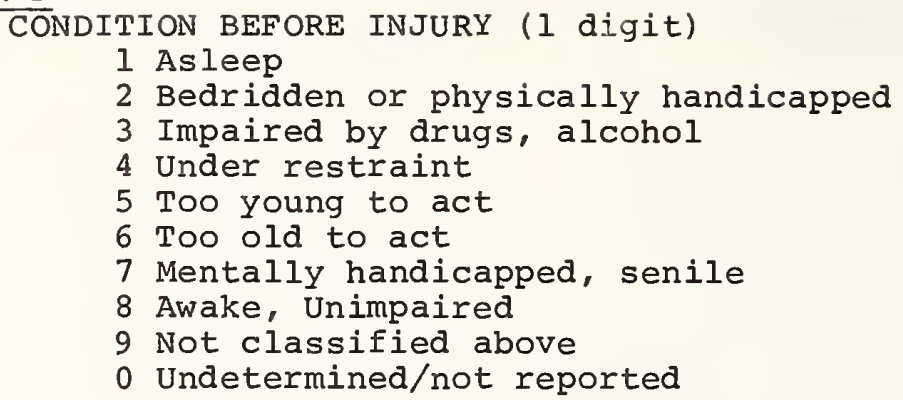

BLOCK Q

WAS THERE A SMOKE DETECTOR PRESENT? IF SO, DID IT OPERATE? (1 digit)

0 Undetermined/not reported

1 Present and operated

2 Present and did not operate

7 Present and operation not reported

8 Not present

\section{BLOCK R}

BLOCK $R$ WILL BE CODED WITH

1-14 letters (left justified blank filled) indicating block of this form for which additional information is provided in NARRATIVE

BLOCK S

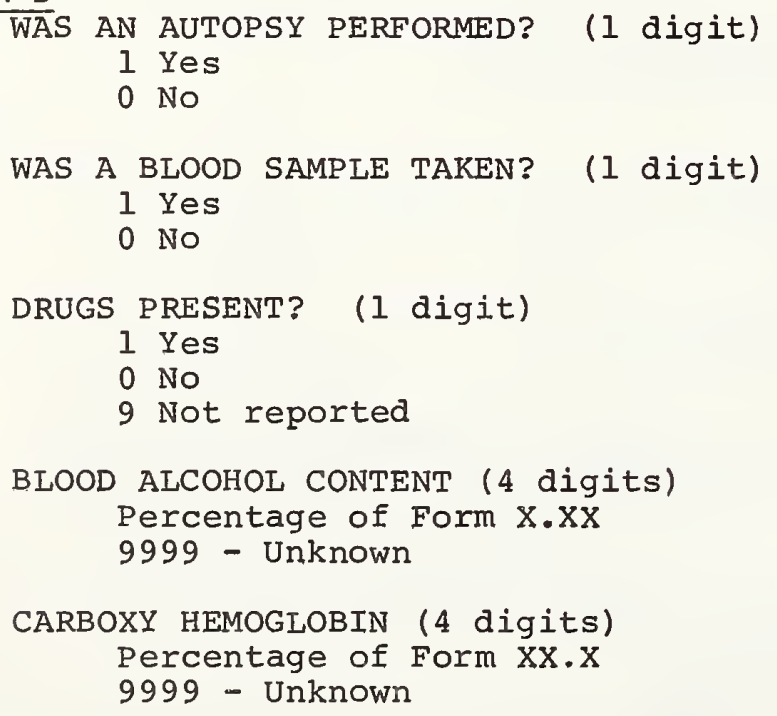

0 No

WAS A BLOOD SAMPLE TAKEN? ( 1 digit)

1 Yes

0 No

DRUGS PRESENT? ( 1 digit)

1 Yes

0 No

9 Not reported

BLOOD ALCOHOL CONTENT (4 digits) Percentage of Form X.XX 9999 - Unknown

CARBOXY HEMOGLOBIN (4 digits) Percentage of Form XX.X 9999 - Unknown

BLOCK T

\section{DATA SOURCES (2 digits)}

For convenience, we use the abbreviation DC for Death Certificate FIR for Fire Incident Report TPC for Telephone/Personal Contact NR for Newspaper Report

o for other 


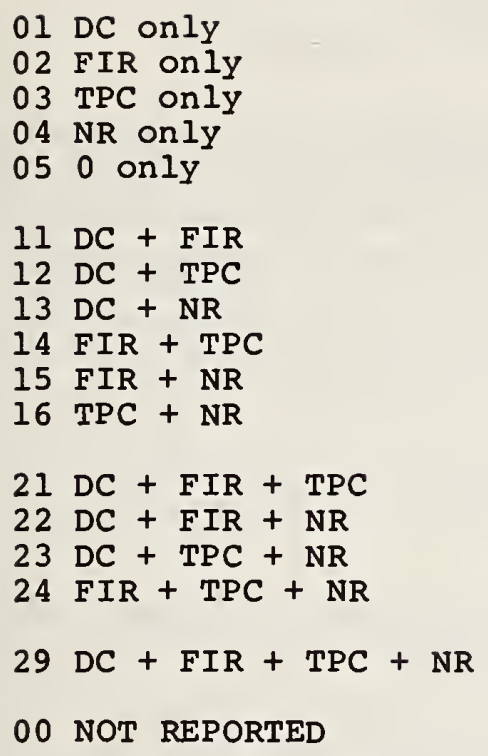

$31 \mathrm{DC}+0$

32 FIR + 0

33 TPC +0

$34 \mathrm{NR}+0$

$41 \mathrm{DC}+\mathrm{FIR}+0$

$42 \mathrm{DC}+\mathrm{TPC}+0$

$43 \mathrm{DC}+\mathrm{NR}+0$

44 FIR + TPC + 0

$45 \mathrm{FIR}+\mathrm{NR}+0$

$46 \mathrm{TPC}+\mathrm{NR}+0$

$51 \mathrm{DC}+\mathrm{FIR}+\mathrm{TPC}+0$

$52 \mathrm{DC}+\mathrm{FIR}+\mathrm{NR}+0$

$53 \mathrm{DC}+\mathrm{TPC}+\mathrm{NR}+0$

54 FIR + TPC + NR + 0

$59 \mathrm{DC}+\mathrm{FIR}+\mathrm{TPC}+\mathrm{NR}+0$ 
DEFINITION OF FIRE "CAUSES"

\begin{tabular}{|c|c|c|c|}
\hline Cause & EQP & $\begin{array}{c}\text { NFPA } 901 \text { Codes } \\
\text { FHI }\end{array}$ & IGF \\
\hline $\begin{array}{l}\text { Incendiary, } \\
\text { Suspicious }\end{array}$ & - & - & $\begin{array}{l}11,12, \\
21,22\end{array}$ \\
\hline $\begin{array}{l}\text { Children } \\
\text { Playing }\end{array}$ & - & - & 36,48 \\
\hline Smoking & - & $30-39$ & - \\
\hline Heating & $10-19$ & - & - \\
\hline Cooking & $20-29$ & - & - \\
\hline $\begin{array}{l}\text { Electrical } \\
\text { Distribution }\end{array}$ & $\begin{aligned} 40 & -49 \\
& -\end{aligned}$ & $28,-54$ & $\overline{-}$ \\
\hline Appliances & $\begin{array}{l}30-39 \\
50=54 \\
56-59\end{array}$ & - & - \\
\hline $\begin{array}{l}\text { Open } \\
\text { Flame }\end{array}$ & 87 & $\begin{array}{l}41-43 \\
44-47 \\
53,55\end{array}$ & $32, \quad 35$ \\
\hline Unknown & & $\begin{array}{l}00,97,99 \\
\text { Non-Numeric }\end{array}$ & \\
\hline Other & & ALL OTHER & \\
\hline
\end{tabular}

EQP : Equipment Involved in Ignition

FHI: Form of Heat of Ignition

IGF : Ignition Factor 
After reviewing available sources of population data, it was found that there appears to be no single definitive approach to calculating populations by population interval. The U.S. Fire Administration has developed population by population interval calculations for the time period in question, but they are for "population protected" not for the population of fatality location needed for this study. While "population protected" is adequate for the upper population intervals $(>50,000)$, it is apparent that considerable discrepancies exist between "population protected" and population of fatality location for population below 50,000 .

Census figures [6] provide 1970 populations by population interval, but include substantial "other urban" and "other rural" categories, which are not defined by population interval.

A method was therefore developed to estimate populations by population interval, using various sources and assumptions, and was used for all states except as noted.

First, the USFA populations for intervals of over 50,000 population were used directly, as was the total population for each state. These were compared with interval totals calculated from Rand McNally Commercial Atlas estimates, and no significant discrepancies were found. An assumption, that the "other rural" category given by 1970 Census totals consisted entirely of areas of under 2500 population, was then made. The Census Supplementary Report PC(S1)-108 [7] was then used to calculate the "other urban" population which belonged in the < 2500 population interval. The 1970 interval of < 2500 was then calculated by summing these "other urban" areas of < 2500 population, all Census places of < 2500, and the "other rural" total. An additional assumption was then made, that rural $(<2500)$ population change in each state between 1970 and 1978 was essentially the same as between 1960 and 1970 (in most cases, a slight decline or little change). This assumption enabled the calculation of total population for the $<2500$ interval for 1978 .

The calculated 1978 populations for intervals $>50,000$ and $<2500$ were subtracted from the 1978 totals, with the remainder falling in intervals between 2500 and 50,000 . The distribution of this remainder among the four intervals in this range was calculated using an additional source, Census documents Series P-25 [8]. The total populations in each of the four intervals between 2500 and 50,000 population as of July 1, 1977 was calculated by adding the populations of individual places given in the Series $\mathrm{P}-25$. The 1978 population total for the $2500-50,000$ range was then distributed among the four intervals within this range in accordance with the distribution calculated for 1977 from the Series P-25.

Two exceptions to the above method of population calculation were made. Wisconsin provided us with a recent census $(1 / 1 / 79)$ of population by population interval, which was used directly. (Wisconsin's population distribution was also. calculated by the described method, and the results were found to be similar to the figures provided to us.) Delaware's population calculation was based entirely on the PC(S1)-108 calculations for populations over 2500 , due to the presence of several large unincorporated areas in the state.

Finally these state population estimates by population interval were totaled for HIGH and LOW states. These estimates, listed in tables 2 and 3 , were used for all subsequent fatality rate calculations.

Given the assumptions which had to be made, and the limited amount of information regarding population intervals that is currently available, there is considerable uncertainty regarding the accuracy of the populations and fatality rates calculated from them. Until better information can be obtained (perhaps from 1980 Census tabulations) these estimates appear to be the best available and are used as such. 
NBS-114A (REV. 2=8C)

\begin{tabular}{|c|c|c|c|}
\hline $\begin{array}{c}\text { U.S. DEPT. OF COMM. } \\
\text { BIBLIOGRAPHIC DATA } \\
\text { SHEET (See instructions) }\end{array}$ & $\begin{array}{c}\text { 1. PUBLICATION OR } \\
\text { REPORT NO. } \\
\text { NBSIR 82-2519 }\end{array}$ & 2. Performing Organ. Report No. & $\begin{array}{c}\text { 3. Publication Date } \\
\text { June } 1982\end{array}$ \\
\hline
\end{tabular}

4. TITLE AND SUBTITLE

RURAI AND NON-RURAL CIVILIAN RESIDENTIAL FIRE FATALITIES IN TWELVE STATES
5. $A \cup T H O R(S)$
A. Gomberg and L. P. Clark

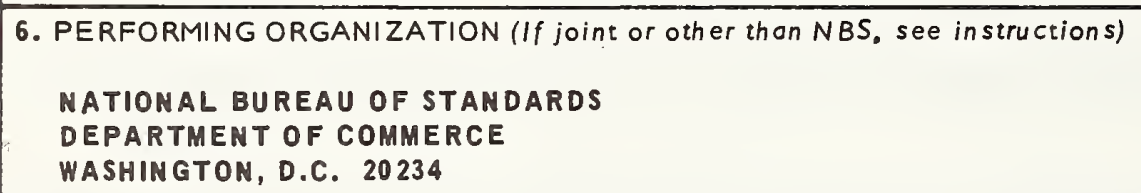

Document describes a computer program; SF-185, FIPS Software Summary, is attached.

11. ABSTRACT (A 200-word or less foctual summory of most significant information. If document includes a significant bibliography or literature survey, mention it here)

The results of an analysis of fire causal factors in over 1600 fire fatalities are presented. The primary emphasis is on the identification of fire causes leading to demonstrated high fatality rates in rural areas. It was found that the most significant rural fire fatality cause was heating equipment, with improper installation and misuse of solid fueled heating equipment predominating. Other fire causes making significant contributions to high rural fatality rates were also investigated and documented. Additional data are currently being collected to enable further evaluation of rural fire problems.

12. KEY WORDS (Six to twelve entries; alphabetical order; copitolize only proper names; and separate key words by semicolons) Residential fires; fire fatalities; fire cause; fire data; fire statistics; heating equipment; rural fires.

13. AVAILABILITY

区. Unlimited

For Official Distribution. Do Not Release to NTIS

$\square$ Order From Superintendent of Documents, U.S. Government Printing Office, WashIngton, D.C. 20402.
14. NO. OF PRINTED PAGES

52

15. Price

$\$ 9.00$

[X] Order From National Technical Information Service (NTIS), Springfield, VA. 2216I 

